\title{
乌ु \\ High-resolution scanning tunneling microscopy investigation of the (12110) and (10000) two-fold symmetric $d$-Al-Ni-Co quasicrystalline surfaces
}

\author{
R. Mäder, ${ }^{1,2, *}$ R. Widmer, ${ }^{1}$ P. Gröning, ${ }^{1}$ S. Deloudi, ${ }^{2}$ W. Steurer,${ }^{2}$ M. Heggen, ${ }^{3}$ P. Schall,,${ }^{3, \uparrow}$ \\ M. Feuerbacher, ${ }^{3}$ and O. Gröning ${ }^{1}$ \\ ${ }^{1}$ Empa, Swiss Federal Laboratories for Materials Testing and Research, nanotech@surfaces Laboratory,, \\ Feuerwerkerstrasse 39, CH-3602 Thun, Switzerland \\ ${ }^{2}$ Laboratory of Crystallography, Department of Materials, ETH Zurich, Wolfgang-Pauli-Strasse 10, CH-8093 Zurich, Switzerland \\ ${ }^{3}$ Institut für Festkörperforschung, Forschungszentrum Jülich, D-52425 Jülich, Germany \\ (Received 25 March 2009; revised manuscript received 27 May 2009; published 30 July 2009)
}

\begin{abstract}
The $d$-Al-Ni-Co quasicrystal exhibits two crystallographically inequivalent twofold symmetric planes: the $\{10000\}$ and $\{12110\}$ surface planes. In particular the twofold symmetric surfaces are very interesting since they are spanned by perpendicular periodic and aperiodic axes. We investigated the (12110) surface of two different compositions $d-\mathrm{Al}_{70} \mathrm{Ni}_{15} \mathrm{Co}_{15}$ and $d-\mathrm{Al}_{72.9} \mathrm{Ni}_{10.4} \mathrm{Co}_{16.7}$ and the (10000) $d-\mathrm{Al}_{72.9} \mathrm{Ni}_{10.4} \mathrm{Co}_{16.7}$ surface by means of low-temperature $(5 \mathrm{~K})$ scanning tunneling microscopy (STM) in ultrahigh vacuum. All three surfaces are characterized with atomic resolution and display large and flat terraces with a columnar structure along the periodic axis. Both compositions of the (12110) $d$-Al-Ni-Co STM investigations revealed that the surface is faceted into $\{12110\}$ and $\{10000\}$ facets with a 1:1 area ratio. The (12110) facets represent the bulk periodicity of $0.4 \mathrm{~nm}$ within the prominent columnar structure which is attributed to a stacking of Al dimers. The sequence of the columnar structure along the aperiodic axis as well as the step heights between the (12110) surfaces could be attributed to the pentagonal tiling edge length of the bulk model. Furthermore, the (12110) surface is identified as one of the densest planes in the bulk model possessing a slightly lower Al concentration as the nominal bulk composition. However, a difference in the fine structure within the columns is observed between the two investigated compositions. The (10000) facet presents an identical surface structure as the unfaceted (10000) surface of the quasicrystal. At the (10000) surface two terraces with different surface structures are identified and compared to the bulk model. One of them shows a bias voltage depending structure. In contrast to the bulk model the minimal observed periodicity on the (10000) surface is doubled to $0.8 \mathrm{~nm}$. On the other hand the aperiodic step height sequence and the order of the columnar motifs along the aperiodic axis are in agreement with the bulk model. The height distribution analysis of the (10000) surfaces exhibits that the surface topmost layer consists of planes which are less dense than the (12110) surface planes.
\end{abstract}

DOI: 10.1103/PhysRevB.80.035433

PACS number(s): 61.44.Br, 68.37.Ef, 68.35.B-

\section{INTRODUCTION}

Quasicrystals (QC) are intermetallic alloys of aperiodic long-range atomic order, which typically exhibit crystallographically forbidden rotational symmetries such as five-, eight-, ten-, or 12-fold. Apart from the intrinsic aperiodic structure and the well known unusual physical bulk properties, ${ }^{1}$ QC possess many interesting surface features such as good oxidation resistance compared to pure $\mathrm{Al}$ single crystals and a particularly low coefficient of friction. ${ }^{2-7}$ In the research of surface properties and their relation to the aperiodic structure the twofold surfaces of the $d$-Al-Ni-Co quasicrystal are of special interest since they allow a simultaneous investigation of a periodic and perpendicular to it an aperiodic axis on same surface. An outstanding example for a simultaneous measurement of surface physical properties directly related to this two inequivalent surface directions of the clean (10000) $d$-Al-Ni-Co surface is the friction anisotropy measurement of Park et al. ${ }^{6}$ They reported a 7.8 times lower friction coefficient along the aperiodic compared to the periodic axis. It can be expected that such anisotropies can be found in more fundamental physical properties such as the electronic local density of states, which can be accessed by scanning tunneling spectroscopy. ${ }^{8}$ The physical interpretation of such measurement will require a detailed knowledge of the atomic arrangement on the surface. Therefore, our paper is concerned with the clarification of the surface structure regarding the local atomic arrangement of the two crystallographically inequivalent twofold (12110) and the (10000) $d$-Al-Ni-Co QC surfaces. One very important question which arises when investigating quasicrystalline surfaces is whether the surface is bulk truncated and reflects therefore bulkstructure related physical properties or if the surface structure undergoes reconstruction. It is a complex task to answer this question, since for a specific crystallographic direction many different inequivalent layers of the QC are potential surface terminations. It is by no means straightforward to determine if there is only one possible termination and to identify it. This can be seen in the case of the most intensely investigated surface structure of the unreconstructed fivefold $i$-Al-Pd-Mn surface, where a number of investigations were performed ${ }^{9}$ but still several issues about the terminating planes remain. ${ }^{10}$ Another interesting question is how would the relation be between a reconstructed quasicrystalline surface and its bulk structure? Can we expect a $\tau$ inflated surface reconstruction, e.g., a $(\tau \times \tau)$ superstructure?

To answer such questions there are two main groups of surface experiments: one group consists of diffraction techniques averaging a large surface area and investigate the long-range order, e.g., spot profile analysis low-energy elec- 
tron diffraction (SPA-LEED), and the other group probing the short-range order on atomic scale, e.g., scanning tunneling microscopy (STM). Both groups have their advantages and can be used complementary to reveal quasicrystalline surface structures. The STM results presented here are therefore compared with the results of surface diffraction methods, ${ }^{11-13}$ STM measurements of the (10000) surface, ${ }^{4,14}$ and are compared in all three dimensions with an appropriated bulk atomic model of the investigated crystals. ${ }^{15}$

The structure of the low-index surface (10000) $d$-Al-Ni-Co was investigated by STM by Kishida et al. ${ }^{14}$ and Park et al. ${ }^{4}$ by helium atom scattering (HAS) and SPALEED (Refs. 11 and 12) by Sharma et al., and by LEED and angle-resolved photoemission spectroscopy (ARPES) by Rotenberg et al. ${ }^{16}$ However, the findings of the local atomic arrangement by STM of Park et al. ${ }^{4}$ and Kishida et al. ${ }^{14}$ were not coherent with each other. In view of the complicated phase diagram ${ }^{17}$ of the Al-Co-Ni system a possible explanation of this discrepancy can reside in the composition of the investigated crystals or differences in the surface preparation procedure. Hence, we performed an extended surface structure investigation of the (10000) $d$-Al-Ni-Co for two different compositions $\mathrm{Al}_{70} \mathrm{Ni}_{15} \mathrm{Co}_{15}$ and $\mathrm{Al}_{72.9} \mathrm{Ni}_{10.4} \mathrm{Co}_{16.7}$. Furthermore, we compared the (10000) surface with a (10000) facet appearing on the (12110) surface of two different orientated crystals of the same composition. We demonstrate here that the (10000) surface possesses two different terminations, the so-called hex and row terraces. These two terrace types show the same characteristic length scales in the periodic and aperiodic directions, but exhibit different surface structure motifs and are assumed to consist of a double bulk layer. The surface structure motifs are then compared with the bulk atomic model and the main surface structure features reveal the origin of the HAS and SPA-LEED diffraction spot.

The low-index (12110) $d$-Al-Ni-Co twofold surface was investigated by Sharma et al. ${ }^{13}$ using HAS and by Rotenberg et al. ${ }^{16}$ using ARPES. The HAS investigation ${ }^{13}$ revealed that the surface is faceted into (12110) and (10000) facets and the (12110) surface showed diffraction peaks which corresponds to the bulk model. Here, we present an extended study of the faceting, the origin of the HAS diffraction spots, the assignment of dense layers as surface termination and a surface structure description in direct space based on a pentagonal tiling deriving from the bulk model with additional information about the chemical composition of the terminating layer.

\section{EXPERIMENTAL PROCEDURES}

In this study we have investigated the twofold symmetric surfaces of three single-grain $d$-Al-Ni-Co QC. The QC differ either in surface orientation or in chemical composition: QC no. 1 with composition $\mathrm{Al}_{70} \mathrm{Ni}_{15} \mathrm{Co}_{15}$ has a (12110) surface orientation, QC no. 2 with the composition $\mathrm{Al}_{72.9} \mathrm{Ni}_{10.4} \mathrm{Co}_{16.7}$ has also a (12110) orientation, and QC no. 3 with the same composition $\mathrm{Al}_{72.9} \mathrm{Ni}_{10.4} \mathrm{Co}_{16.7}$ as QC no. 2 possesses a (10000) surface orientation. QC no. 1 was grown by the Bridgman method, whereas QC no. 2 and QC no. 3 were grown by means of the self-flux technique. ${ }^{18,19}$ In the latter, the high-purity starting materials were sealed in a tip-shaped crucible in the following proportions: Al: 77.0 at.\%, Co: 12.5 at.\%, and Ni: 10.5 at.\%. The crucible was placed in a chamber furnace and the temperature profile consisted in slow cooling of the melt by $0.6^{\circ} / \mathrm{h}$ between 1373 and $1283 \mathrm{~K}$. At $1283 \mathrm{~K}$ the residual melt was decanted from the grown crystals. The final composition of the single QC was determined as $\mathrm{Al}: 72.9$ at.\%, $\mathrm{Co}: 16.7$ at.\%, and $\mathrm{Ni}: 10.4$ at.\% by means of inductively coupled plasma optical emission spectroscopy.

All three QC were oriented using Laue diffraction and cut to expose the desired crystallographic surface. The surfaces were then mechanically polished to mirror finish with diamond paste down to a grain size of $0.1 \mu \mathrm{m}$. The following UHV preparation (base pressure below $10^{-10}$ mbar) was carried out by repeated sputter-annealing cycles. The cycles consisted of $\mathrm{Ar}^{+}$-ion bombardment at $1.5 \mathrm{kV}$ at grazing angle of $30^{\circ}$ for $30 \mathrm{~min}$ followed by annealing to $1073 \mathrm{~K}$ for $1 \mathrm{~h}$, as monitored by an optical pyrometer Raytek MA2B with the emissivity set to $\varepsilon=0.35$. This procedure was repeated six to eight times. The as-prepared surfaces showed sharp LEED patterns from 15 to $170 \mathrm{eV}$ kinetic electron energy. The subsequent STM measurements were carried out using a commercial UHV Omicron low-temperature STM cooled down with liquid nitrogen (at $77 \mathrm{~K}$ ) or liquid helium (at $5 \mathrm{~K}$ ). Mechanically cut $\mathrm{Pt}_{80} / \mathrm{Ir}_{20}$ tips were used for the STM imaging and the recorded STM data were further processed by the WSXM software. ${ }^{20}$ The X-ray photoelectron spectroscopy (XPS) investigations have been carried out in an Omicron ESCA system equipped with a VSW 125 HR electron analyzer operating at a base pressure of $5 \times 10^{-11}$ mbar. A nonmonochromatized Al K $\alpha$ x-ray source of $1486.6 \mathrm{eV}$ photon energy was employed for the presented XPS measurement. After the UHV-STM investigations, QC no. 1 and QC no. 2 have been characterized by single-crystal $\mathrm{X}$-ray diffraction (XRD) analog to former XRD studies of $d$-Al-Ni-Co. ${ }^{21}$ The XRD images of the QC no. $1\left(\mathrm{Al}_{70} \mathrm{Ni}_{15} \mathrm{Co}_{15}\right)$ composition is comparable to Fig. 3(d) in Katrych and Steurer ${ }^{21}$ and shows diffuse scattering around the Bragg reflections. Therefore, we assumed that the investigated QC no. 1 consists of nanodomains of the basic cobalt-rich phase, the S1 phase, and maybe of some approximant domains. On the other hand, the XRD images of the QC no. $2\left(\mathrm{Al}_{72.9} \mathrm{Ni}_{10.4} \mathrm{Co}_{16.7}\right)$ showed hardly any diffused scattering and the bulk structure could be assigned to a well ordered basic cobalt-rich $d$-Al-Ni-Co single phase comparable to Fig. 3(a) in Katrych and Steurer. $^{21}$

\section{RESULTS AND DISCUSSION}

In the following the structures observed by STM are compared and discussed using the Co-rich $d$-Al-Ni-Co bulk structure model of Deloudi. ${ }^{15}$ According to the classification of the bulk structure for QC no. 2 and QC no. 3 by XRD this structure model is found to be appropriate. The bulk structure consists of periodically stacked planes with an aperiodic atomic arrangement within the planes. Figure 1(a) schematically illustrates the model. One period consists of four inequivalent aperiodic layers A, B, C, and D which are separated by $0.20 \mathrm{~nm}$ and stacked along the [00001] direction, 

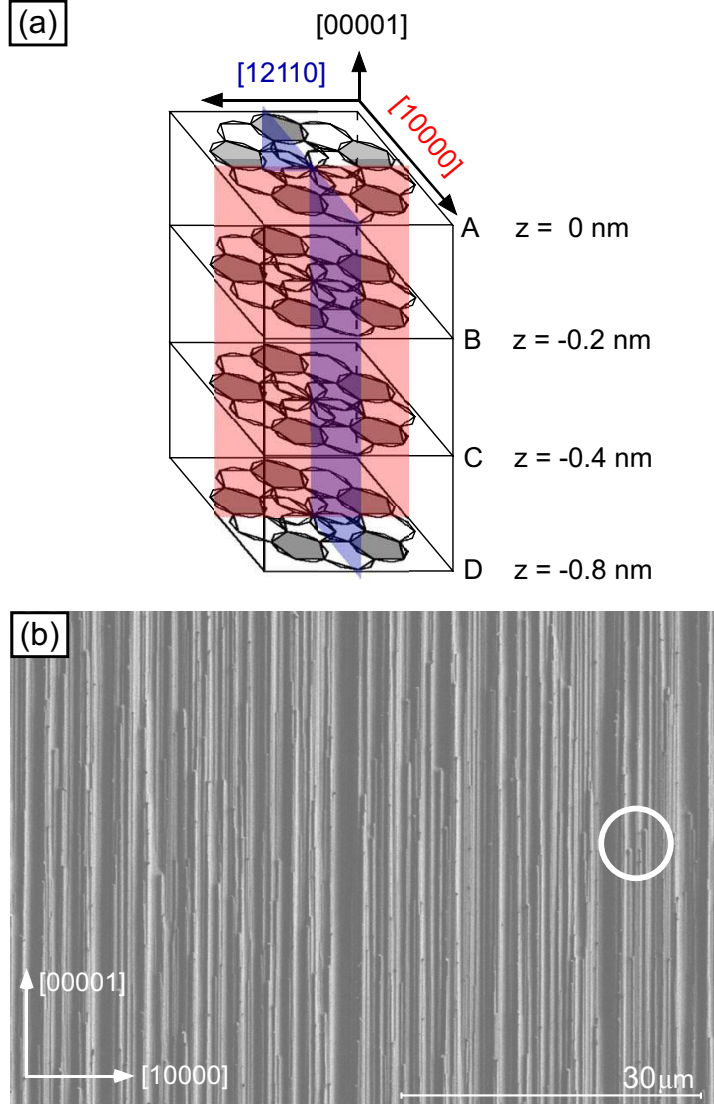

FIG. 1. (Color online) (a) Sketch of the applied bulk model. It shows the layer structure of the $d$-Al-Ni-Co quasicrystal. Each layer A, B, C, and D possesses fivefold rotational symmetry with a 0.2 $\mathrm{nm}$ interlayer distance. The main axes are shown: the periodic socalled tenfold [00001] and the two so-called twofold axes [10000], [12110] with their corresponding planes. (b) SEM image of the twofold (12110) surface of the $d-\mathrm{Al}_{70} \mathrm{Ni}_{15} \mathrm{Co}_{15}$ (QC no. 1) shows the prominent columnar structure motif along the tenfold axis. The white circle highlights an area of column endings.

the tenfold axis. The periodic repetition of layers leads to a columnar structure along the [00001] direction. The aperiodic long-range order is obtained by decorating the vertices of a Pentagonal-Penrose tiling with two different $\sim 20 \AA$ diameter clusters in an antiparallel orientation. ${ }^{15}$ Cutting the $d$-Al-Ni-Co along the [00001] direction yields, expressed in the indexing scheme of Steurer, ${ }^{22}$ two inequivalent low-index crystallographic surfaces the (10000) and orthogonal to it the (12110) which is equivalent to the (001-10) surface. Due to the tenfold rotation symmetry the smallest angle between the $\{10000\}$ and $\{12110\}$ planes is $18^{\circ}$. Additionally, it should be noticed that the Bragg spots of the (12110) planes obtained from the Deloudi model shows the same pattern as the socalled (D2) electron diffraction pattern used earlier in the literature. ${ }^{23-25}$

After UHV surface preparation the surface structure on the microscopic level was investigated using scanning electron microscopy (SEM). The sample was transferred under ambient conditions from the UHV chamber to the SEM instrument, which, however, does not induce relevant structural changes on the micrometer level of interest here. Figure

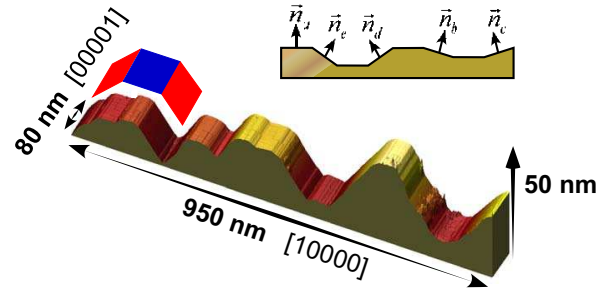

FIG. 2. (Color online) 3D STM image representation of the (12110) $d-\mathrm{Al}_{72.9} \mathrm{Co}_{16.7} \mathrm{Ni}_{10.4}$ (QC no. 2) surface. This large scan shows the faceting of the surface along the aperiodic direction [10000], straight or blue for the (12110), and inclined or red for the (10000) facet, respectively. The columnar structure feature is also prominent along the periodic tenfold direction $(80 \mathrm{~nm})$. The inset in the upper right corner shows schematically the five different observed facets: $+36^{\circ},+18^{\circ}, 0^{\circ},-18^{\circ},+18^{\circ}$, and $-36^{\circ}$.

1(b) shows a large area $120 \times 60 \mu \mathrm{m}^{2}$ SEM image of the twofold (12110) QC no. 1 surface. The SEM image reveals a strong asymmetry between the periodic tenfold axis [00001] and the aperiodic [10000] direction. This asymmetry is an intrinsic property of the decagonal bulk structure and manifests itself on this macroscopic scale by forming out a columnar structure along the [00001] axis. Although, the columns observed in the SEM have a high aspect ratio they are not infinitely long (a few $\mu \mathrm{m}$ ) and abrupt terminations of these columns can be observed. A spot with three such terminations is highlighted by a white circle in Fig. 1(b). As we shall see similar terminations can be observed in the STM on the nanoscopic level, which can be attributed to bunched atomic steps.

One advantage of the STM experiment in contrast to SEM is that STM provides quantitative information about the surface topography. Figure 2 displays a 3D representation of a (12110) surface STM image of QC no. 2, which shows that the surface is faceted. A detailed analysis reveals that the surface is faceted into $\{12110\}$ planes parallel to the macroscopic surface (or in some very rare cases $\pm 36^{\circ}$ ) and into $\{10000\}$ planes with an angle of $\pm 80^{\circ}$ to the macroscopic surface. Although the faceting of the (12110) surface was already evidenced by ion scattering ${ }^{13}$ some additional information can be given on the facet width and occurrence; see Table I. It first shows that the net orientation of the surface is conserved and second from the equilibrium morphology problem addressed by Herring ${ }^{26}$ we also conclude, as Sharma et $a l .{ }^{13}$ that the total surface tension is reduced by breakup of the surface into new orientations:

$$
\begin{gathered}
A_{\vec{n}_{a}} \vec{n}_{a}=\sum_{i} A_{\vec{n}_{i}} \vec{n}_{i}, \\
A_{\vec{n}_{a}} \gamma\left(\vec{n}_{a}\right)>\sum_{i} A_{\vec{n}_{i}} \gamma_{i}\left(\vec{n}_{i}\right),
\end{gathered}
$$

where $\gamma$ is the surface tension and $A_{\vec{n}_{i}}$ is the area of the surface of orientation $\vec{n}_{i}$. From this argument one can deduce that the surface tension $\gamma$ is lower for the $\{10000\}$ than for the $\{12110\}$ planes. Table I indicates also that the width of the $\{10000\}$ and $\{12110\}$ planes are equally distributed and the widths of both planes are large enough for a detailed 
TABLE I. Analysis of the $d$ - $\mathrm{Al}_{72.9} \mathrm{Co}_{16.7} \mathrm{Ni}_{10.4}$ QC no. 2 (12110) $d$-Al-Ni-Co surface faceting over a $2 \mu \mathrm{m}$ large surface section.

\begin{tabular}{lcccccc}
\hline \hline Facet type & $n_{d}$ & $n_{c}$ & $n_{a}$ & $n_{b}$ & $n_{e}$ & Nonspecific \\
Angle (deg) & $-36^{\circ}$ & $-18^{\circ}$ & $0^{\circ}$ & $+18^{\circ}$ & $+36^{\circ}$ & \\
Equivalent plane & $\{12110\}$ & $\{10000\}$ & $\{12110\}$ & $\{10000\}$ & $\{12110\}$ & \\
Total facet width (\%) & 0.5 & 20.4 & 49.8 & 24.8 & 0.5 & 1.5 \\
Facet mean width (nm) & 5 & 23.5 & 9.5 & 11.7 & 5 & 7.6 \\
Facet standard deviation (nm) & 2.2 & 30.2 & 16.6 & 14.3 & 2.2 & 3.6 \\
\hline \hline
\end{tabular}

surface investigation. The STM images presented in the further course of this work will prove that both facet types of the surface show different atomic structures and are therefore good candidates to be investigated and to compare the surface with the introduced bulk model. The following discussion is organized as follows: first we will concentrate on the structural investigation of the (12110) surface and compare the results with the bulk model. In the second part of the paper the results of the (10000) surfaces analysis are presented and compared with the bulk model. In the last part the main differences of the surface structure studies are discussed.

\section{A. Surface structure of (12110) $d$-Al-Ni-Co}

Similar to the terminations of the columnar features shown in Fig. 1(b), Fig. 3(a) presents on a smaller scale an STM image of an area of ending columns. This region is highly stepped and shows a large number of parallel well defined atomic planes. A general finding for both twofold $d$-Al-Ni-Co surfaces is that the steps between well defined atomic planes are straight and oriented exactly along the tenfold [00001] axis. $^{14}$ The length of the atomically flat terraces along the periodic [00001] tenfold axis is at least several hundreds of nanometers whereas the maximum width of the terraces along the aperiodic twofold direction [10000] is about $100 \mathrm{~nm}$. We like to emphasize here that the terraces typically extend very long in the [00001] direction and that such regions of terrace terminations are rather rarely observed. The sequence of the terrace step height provides information about the layer succession along the surface normal, i.e., the [12110] direction. Figure 3(b) displays the STM height profile taken along the black dashed line in Fig. 3(a). The vertical lines and the $L$ and $S$ bars on the right-hand side of Fig. 3(b) illustrate that the sequence of the terrace step heights can be described by a part of the Fibonacci sequence. Experimentally, we identified the Fibonacci building units with $S=0.220(7) \mathrm{nm}$ and $L=0.374(2) \mathrm{nm} \approx \tau * S$, respectively (with $\tau=1.61806 \ldots$ being the value of the golden mean). The resulting fragment of the Fibonacci sequence SLLSLSLLSL indicates the aperiodic stacking of the terraces in [12110] direction. Moreover, the line profile displays the topographic roughness of these terraces $(0.14 \mathrm{~nm} \mathrm{rms}$ and $\Delta z=0.20 \mathrm{~nm})$. This surface corrugation is typical for a QC compared, e.g., with $\Delta z=0.15 \mathrm{~nm}$ of the fivefold Al-Pd-Mn surface ${ }^{27}$ but rather large compared with a corrugation of less than $0.05 \mathrm{~nm}$ for the Al (111) surface. ${ }^{27}$
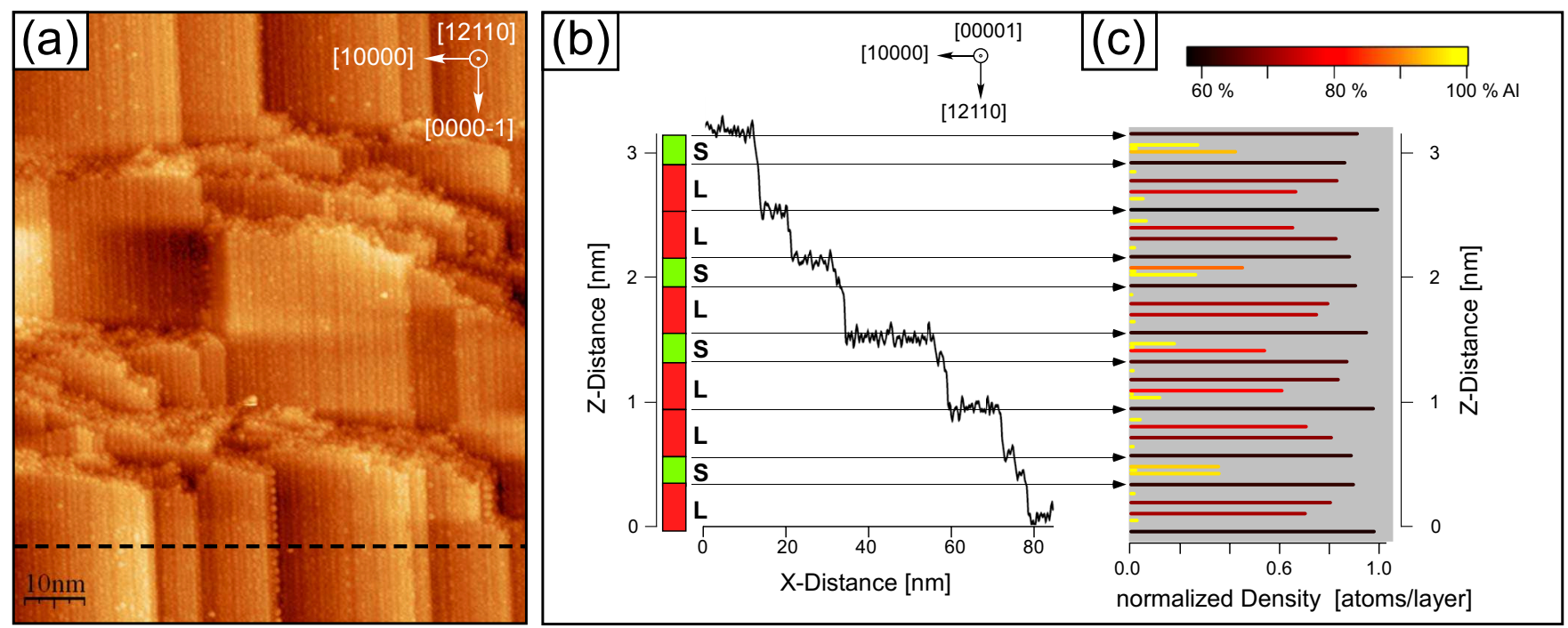

FIG. 3. (Color online) (a) STM image of the (12110) $d-\mathrm{Al}_{72.9} \mathrm{Ni}_{10.4} \mathrm{Co}_{16.7}(\mathrm{QC}$ no. 2) surface recorded at $5 \mathrm{~K}$, window size 84 $\times 100 \mathrm{~nm}^{2}, \Delta z=8.3 \mathrm{~nm}, U_{T}=-0.5 \mathrm{~V}, I_{T}=50 \mathrm{pA}$, it shows a strongly terraced area with columnar endings along the tenfold direction. (b) Height profile recorded along the dashed black line in (a). The according aperiodic step height sequence is indicated. (c) shows the normalized density of planes of the bulk model along the [12110] axis whereas the color code (color online) is the calculated composition of each plane. 
TABLE II. Analysis of the step heights sequences of (12110) surfaces for both compositions.

\begin{tabular}{lccc}
\hline \hline $\begin{array}{l}\text { Averaged } \\
\text { measured } \\
\text { steps heights } \\
(\mathrm{nm})\end{array}$ & Occurrence & $\begin{array}{c}\text { Plane distance } \\
\text { between densest } \\
\text { planes from } \\
\text { model }(\mathrm{nm})\end{array}$ & $\begin{array}{c}\text { Fibonacci } \\
\text { sequence }\end{array}$ \\
\hline $0.220 \pm 0.007$ & 12 & 0.234 & $S$ \\
$0.374 \pm 0.002$ & 15 & 0.378 & $L$ \\
$0.609 \pm 0.003$ & 6 & 0.611 & $L S$ \\
1.01 & 1 & 0.989 & LSL \\
& 0 & 1.601 & LSLLS \\
2.54 & 1 & 2.590 & LSLLSLSL \\
\hline \hline
\end{tabular}

To investigate the sequence of terrace steps more extensively all measured step heights (in total 35) of both compositions $\mathrm{Al}_{72.9} \mathrm{Ni}_{10.4} \mathrm{Co}_{16.7}$ (30 steps) and $\mathrm{Al}_{70} \mathrm{Ni}_{15} \mathrm{Co}_{15}$ (five different step heights) were analyzed. No significant differences could be detected between the two different compositions. The 35 step heights agree well to the progression of the $S=0.220 \mathrm{~nm}$ Fibonacci series, as shown in Table II. It can be observed that the frequency is much higher for the zeroth generation $S$ and the first generation $L$ (with 12 and 15 times) and that the step heights corresponding to subsequent generations are very rarely observed. The third column lists the values for the interplane distances of the densest planes in the used model structure, which we connect with the steps and which we try to identify in the following. Figure 3(c) displays a section of the succession of planes in the bulk model along the [12110] axis. The color code used in Fig. 3 (c) stands for the composition of each plane ranging from black [60\% $\mathrm{Al}$ and $40 \%$ transition metal (TM) atoms] to bright $(100 \% \mathrm{Al}$ atoms). The horizontal axis in Fig. 3(c) indicates the density of the (12110) bulk planes which are normalized by the maximum value of the densest planes which is 10.32 atoms $\mathrm{nm}^{-2}$. This maximum density for a single plane is significantly higher (about $150 \%$ ) compared to the averaged density of one of the two planes which represent the termination of the fivefold $i$-Al-Pd-Mn surface. ${ }^{10}$ However, a single (111) bulk layer of the Al crystal with a density of 14.08 atoms $\mathrm{nm}^{-2}$ is denser than the densest (12110) bulk planes of the $d$-Al-Ni-Co, the volumetric density is of course similar in each case. The arrows denote the experimental terrace step heights in Fig. 3(b) and point to the planes of highest density in Fig. 3(c). It is obvious that the sequence of the experimental terrace step heights agrees well with the sequence of the densest planes in the bulk model. An analogous result to find the surface termination of the $i$-Al-Pd-Mn fivefold surface was found by selecting two closely spaced planes rising in a dense termination. ${ }^{10}$ These so-called plane families possess also the same sequence as the measured step heights. The selection of the sequence of the (12110) $d$-Al-Ni-Co densest planes is justified by the fact that the STM images of the $\{12110\}$ planes appear as a dense plane with a low corrugation. In fact the rms of the measured surface corrugation is by a factor of 2 lower than the smallest plane distances between two densest (12110) $d$-Al-Ni-Co bulk planes. Also the analysis of the height distribution of single terraces shows a uniform single Gaussian distribution which leads to the assumption that no surface steps are involved within one $\{12110\}$ plane. Additionally, the analysis of the model shown in Fig. 3(c) exhibits a correlation between the densities of the $\{12110\}$ planes and their composition. These observations indicate that the $\{12110\}$ terraces show along the [12110] axis the same aperiodic sequence as the bulk model and possess a chemical composition which is low in $\mathrm{Al}$, with $61 \% \mathrm{Al}$ and $39 \% \mathrm{TM}$.

In contrast to the discussion above where both compositions $\mathrm{Al}_{70} \mathrm{Co}_{15} \mathrm{Ni}_{15}\left(\mathrm{QC}\right.$ no. 1) and $\mathrm{Al}_{72.9} \mathrm{Co}_{16.7} \mathrm{Ni}_{10.4}$ (QC no. 2) could be treated together as they showed the same aperiodic sequence along the [12110] axis, the following discussion of the structural details on the (12110) terraces has to be split in two parts due to differences in the surface structure between the two compositions. Since the XRD identified the QC no. 2 composition as a well ordered basic cobalt-rich $d$-Al-Ni-Co single phase we consider this phase as the model phase in this paper. Therefore, we discuss first in detail QC no. 2 and use a Co-rich structure model and elucidate then only the major differences of the surface structure of phase QC no. 1. The STM images of both compositions show only a very weak influence of the sample bias voltage to the surface structure which can be neglected in the following discussion. Figure 4(a) shows a representative high-resolution STM image of QC no. 2. As mentioned, also on a very small scale the surface structure is dominated by columnar features along the [00001] direction. These columnar features are denoted by small black rectangles in the lower part of Fig. 4(a). The repetition period of structural features along the [00001] direction in Fig. 4(a) is illustrated in Fig. 4(b) by a row-byrow one-dimensional (1D) fast Fourier transform (FFT). One can readily identify in the FFT image the columns with a pronounced experimental $0.38(2) \mathrm{nm}$ periodicity which corresponds within the error of measurement to the bulk periodicity of $0.408 \mathrm{~nm} .{ }^{28}$ Apart from the $0.4 \mathrm{~nm}$ periodic columnar structure around $75 \%$ of the surface area possess periodicities larger than $0.4 \mathrm{~nm}$, such as $0.8,1.2$, or even 1.6 $\mathrm{nm}$. Nevertheless, the 1D FFT reveals a relation of these complex regions with the bulk structure as their periodicity is a multiple integer of $0.4 \mathrm{~nm}$. We might therefore consider these regions as complex surface reconstructions commensurate with the bulk. The upper left panel in Fig. 4(a) shows such a reconstruction with different periodicities where bended structures larger than $1 \mathrm{~nm}$ and oriented along the tenfold axis are present. On the other hand the upper right panel displays two well defined columns of $0.4 \mathrm{~nm}$ periodicity which are characteristic features as can be seen from Fig. 4(a). It is obvious that these columns consist of small elliptical features having the long axis in the [10000] direction of $0.75-1.0 \mathrm{~nm}$ length and the short axis in the [00001] direction of about $0.3 \mathrm{~nm}$ width. Based on the aspect ratio and the height profile along the ellipses we assume a dimer structure to be the origin of this structure with a distance of about $0.25 \mathrm{~nm}$ between the two atoms. These dimers are indicated by four pairs of gray dots separated by $0.25 \mathrm{~nm}$ (along the [10000] direction). In comparison $0.2456 \mathrm{~nm}$ is the nearest in-plane distance of atoms encountered in the bulk model. ${ }^{28}$ This distance is found between the center and the edge atom of the smallest pentagon. The aperiodic se- 


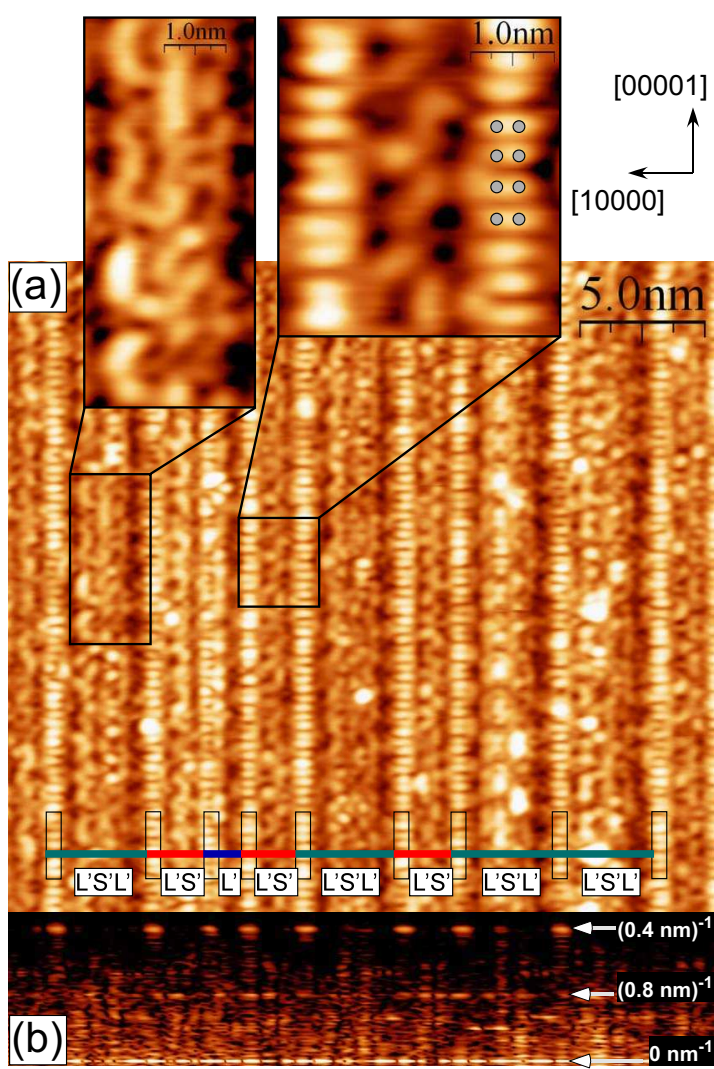

FIG. 4. (Color online) Detailed structure analysis of the (12110) $\mathrm{Al}_{72.9} \mathrm{Co}_{16.7} \mathrm{Ni}_{10.4}$ (QC no. 2) surface. (a) High-resolution STM image, window size $28 \times 26 \mathrm{~nm}^{2}, \Delta z=0.268 \mathrm{~nm}, V=-0.5 \mathrm{~V}$, and $I_{T}=70 \mathrm{pA}$, recorded at $5 \mathrm{~K}$. The very prominent columnar structures are indicated by black rectangles. Its aperiodic sequence is illustrated by colored boxes. (b) The 1D row-by-row FFT image of (a) along the tenfold axis. The columnar structure shows strong reflections at $0.4 \mathrm{~nm}$ and weaker ones at $0.8 \mathrm{~nm}$. The area in between the columnar structure exhibits more faint reflections whereas some of the 0.4 and $0.8 \mathrm{~nm}$ reflection spots are still present.

quence of the main structure feature, the $0.4 \mathrm{~nm}$ periodic columnar structure, along the [10000] direction is indicated in the lower part of Fig. 4(a) by colored bars. A large number of distances between these columns along [10000] direction have been analyzed. The averaged separation with $L^{\prime}$ $=1.48 \mathrm{~nm}$ and $L^{\prime} S^{\prime}=2.24 \mathrm{~nm}$ fits well into a Fibonacci sequence. Here the prime denotes the difference to the Fibonacci building blocks of the step height sequence as shown in Fig. 3(b).

Despite the difference in the XRD patterns and consequently the atomic bulk structure, the step heights between $\{12110\}$ terraces of QC no. 1 and QC no. 2 were shown to be identical. The (12110) surface structure of QC no. 1 presented in Fig. 5(a) exhibits also structure units resembling QC no. 2, For instance the $0.38(2) \mathrm{nm}$ periodic columnar structures present bright spots in the FFT image in Fig. 5(c). But in contrast to Fig. 4(b), the FFT image Fig. 5(c) reveals groups of pairs instead of single spots at $(0.4 \mathrm{~nm})^{-1}$ at the position of one column. This pronounced difference is best observed in real space at the enlarged columnar structure shown in Fig. 5(b). In contrast to the resembling columns of

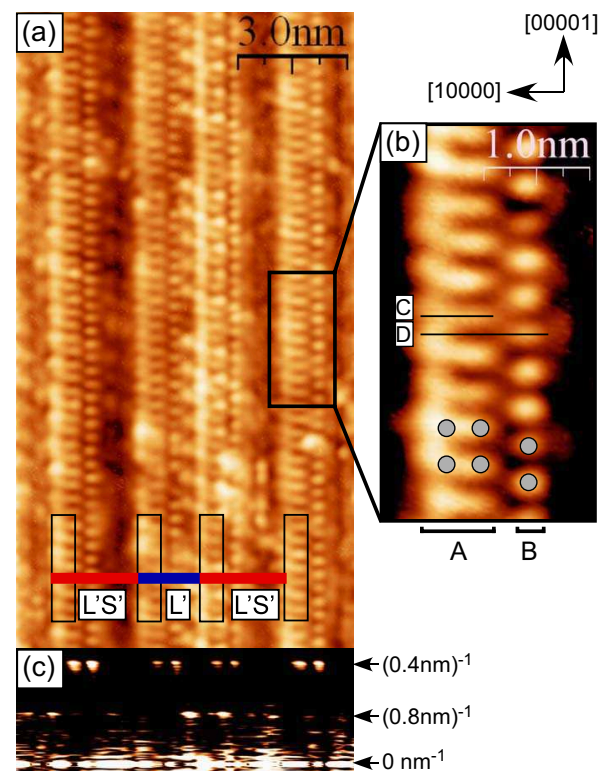

FIG. 5. (Color online) Detailed structure analysis of the (12110) $\mathrm{Al}_{70} \mathrm{Ni}_{15} \mathrm{Co}_{15}$ (QC no. 1) surface. (a) High-resolution STM image, window size $9 \times 18 \mathrm{~nm}^{2}, \Delta z=0.112 \mathrm{~nm}, V=0.264 \mathrm{~V}$, and $I_{T}$ $=50 \mathrm{pA}$ recorded at $5 \mathrm{~K}$. Similar to Fig. 4 , the columnar pattern is surrounded by black rectangles. Its aperiodic sequence is indicated by colored boxes. (b) The enlargement shows the columnar motif of the $\mathrm{Al}_{70} \mathrm{Co}_{15} \mathrm{Ni}_{15}$ (QC no. 1) surface which is slightly different to (QC no. 2). The main difference is the additional row B showing one bright spot which is shifted downward with respect to the A row by $0.2 \mathrm{~nm}$. This is illustrated by the lines $C$ and D. (c) shows the 1D row-by-row FFT image of (a) along the tenfold axis. The columnar structure shows strong reflections at $0.4 \mathrm{~nm}$ and weaker ones at 0.8 $\mathrm{nm}$. In this representation the FFT shows hardly any reflections for the complex structured area.

QC no. 2 which are labeled by the letter A we see an additional row denoted with $\mathrm{B}$. This $\mathrm{B}$ row consists of round features with a $0.3 \mathrm{~nm}$ diameter which might be best regarded as a row of single atoms. The vertical shifts between the $\mathrm{A}$ and $\mathrm{B}$ rows of $0.2 \mathrm{~nm}$ along the [00001] axis are denoted with the lines $C$ and D in Fig. 5(b). This $0.2 \mathrm{~nm}$ distance can be assigned to the interlayer distance along the [00001] axis of the bulk model. The aperiodic distribution of the columns along the [10000] axis follows the same Fibonacci sequence as used to described the one of QC no. 2. A more quantitative approach to describe the aperiodic sequence of the columns is made in Table III. It lists the arithmetic mean value and the frequency of the Fibonacci lengths between the columns which is independent of the composition. It reveals that only three different segments lengths between the columns are observed with the most prominent segment length being the $L^{\prime} S^{\prime}=2.24 \mathrm{~nm}$ Fibonacci unit. Next to the additional single atom row B, a second prominent difference in the surface structure of QC no. 2 is observed within the area between the columns. The structure there is not as complex as in the case for QC no. 2 and hardly any curved structures are visible. Some weak $0.8 \mathrm{~nm}$ periodic oscillations are present in the FFT in Fig. 5(c) but in general the surface structures between the columns appear to be lower in density in the case of QC no. 2. We like to stress 
TABLE III. Analysis of the aperiodic distribution of the columnar structure features along the [10000] direction compared to the bulk model.

\begin{tabular}{lccc}
\hline \hline $\begin{array}{l}\text { Distances between } \\
\text { columns STM } \\
(\mathrm{nm})\end{array}$ & Occurrence & $\begin{array}{c}\text { From tiling: } \\
1.979 \mathrm{~nm} \\
2 \tau^{n} \cos \left(18^{\circ}\right)\end{array}$ & $\begin{array}{c}\text { Fibonacci } \\
\text { sequence }\end{array}$ \\
\hline & & $n=-3$ & $S^{\prime}$ \\
$1.48 \pm 0.01$ & 13 & $n=-2$ & $L^{\prime}$ \\
$2.24 \pm 0.13$ & 28 & $n=-1$ & $L^{\prime} S^{\prime}$ \\
$3.95 \pm 0.12$ & 11 & $n=0$ & $L^{\prime} S^{\prime} L^{\prime}$ \\
\hline \hline
\end{tabular}

that this observation holds for many different scan areas of the two surfaces with each time new surface preparation.

The investigation of the two different compositions (and phases) QC no. 1 and QC no. 2 shows slightly different surface structures. Apart from that, it seems that both surfaces have the same underlying "framework." We can shown that in all three high symmetry directions both compositions agree, in their step heights [12110], periodicity within the columns [00001] and aperiodic distribution of the columns [10000]. This framework is indeed identical also for the appropriated bulk structure models for the composition of our QC no. 1 (Ref. 28) and QC no. $2 .{ }^{15}$ Next to the different periodicity and small changes in atomic positions, both structure models use the same interlayer distances of $0.20 \mathrm{~nm}$ along the periodic direction [00001] and use the same tiling edge length of $1.979 \mathrm{~nm}$ within the aperiodic layers. In the following we attempt to position the terminating planes and the columns with respect to the fivefold symmetric tiling by comparing in detail the (12110) surface structure with the bulk structure. Figure 6(a) shows a section of Fig. 4(a) where again the dimer columns are denoted by black rectangles and the corresponding intercolumnar distances by colored rectangles. The black arrows indicate rows of topographic depression at the surface. Figure 6(b) presents a simple ball model of the (12110) surface. It displays one representative dense plane of the bulk model taken along the yellow stripe in Fig. 6(c). The centers of the atom positions in Fig. 6(b) are denoted by color, violet for $\mathrm{Al}$ and red for TM atoms, respectively. The ratio of the atomic radius with $r(\mathrm{Al}) / r(\mathrm{TM})$ $=1.6$ was chosen to fit best the STM image shown in Fig. 6(a). Figure 6(c) displays the tenfold symmetric (00001) view of the Co-rich bulk model. ${ }^{15}$ In this representation the (12110) surfaces are horizontal cuts through the model. The superimposed pink pentagonal Penrose tiling has an edge length of $1.979 \mathrm{~nm}$ where the vertices are decorated by the so-called wheel-like motifs. ${ }^{28}$ Six of them are marked with blue circles possessing a $1.22 \mathrm{~nm}$ diameter. These wheel-like motifs are surrounded by ten small decagonal motifs indicated by red circles with $0.49 \mathrm{~nm}$ diameter, where each of them has a center atom. Since the densest planes of the bulk model are located at the vertices of the superimposed tiling the measured step heights between the (12110) terraces can be directly identified as the distances between the centers of the $0.49 \mathrm{~nm}$ small red motifs, along the [12110] axis, which is indicated on the right-hand side of Fig. 6(c). For discussing the positions of the structural features of the (12110)

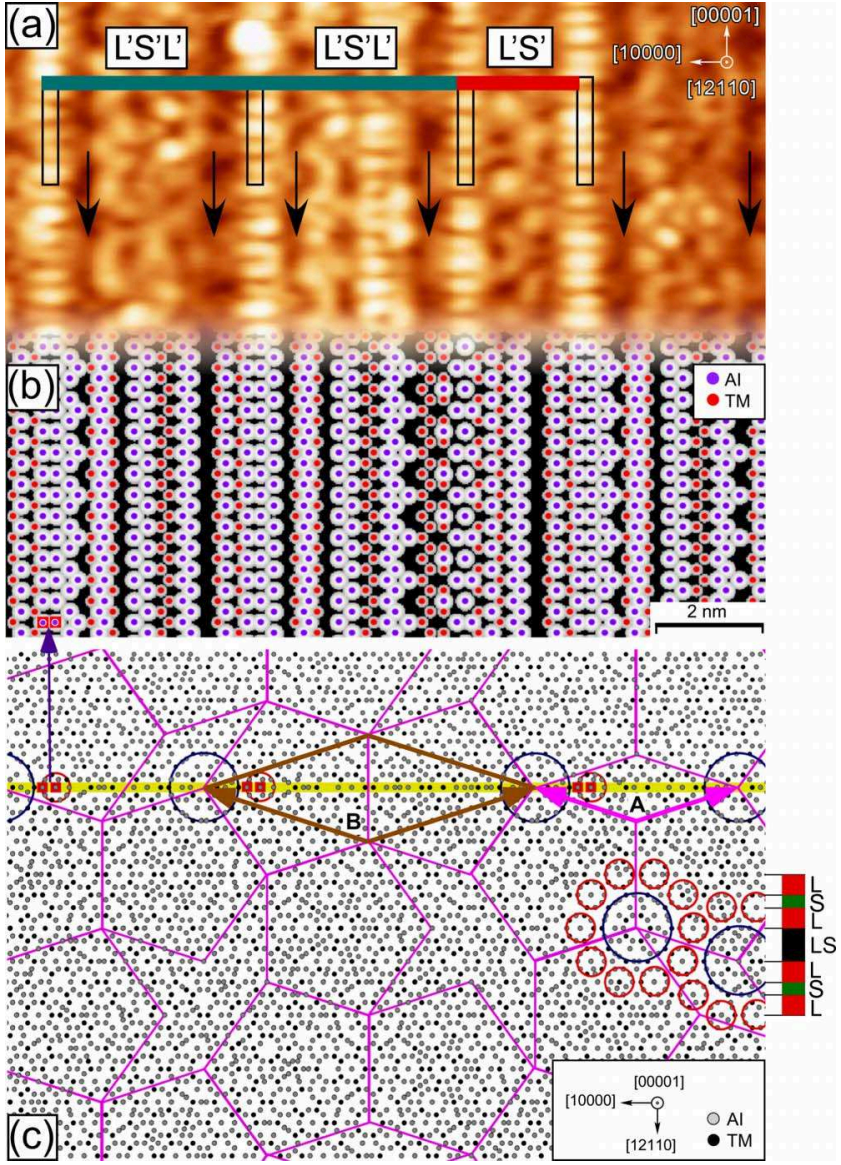

FIG. 6. (Color online) Comparison study of the (12110) surface structure with the bulk model. (a) STM image, a section out of Fig. 4 black rectangles denotes the columns and the black arrows indicate rows of lower atom density. (b) Modeling of a representative bulk model of the (12110) densest planes taken along the yellow stripe in (c). (c) Tenfold view of the Co-rich Al-Ni-Co bulk model decorated with a $1.979 \mathrm{~nm}$ edge length pentagonal Penrose tiling.

surface within the densest planes of the bulk structure it is the best to consider the columnar structure indicated by the black rectangles in Fig. 6(a). As discussed above the elliptic structure is assumed to be formed by two nearestneighboring atoms separated by $0.2456 \mathrm{~nm} .{ }^{28}$ Figure $6(\mathrm{~b})$, which represents a dense plane of the bulk structure model, shows such dimers at the positions of the columns at the surface. These rows of dimers consist of $0.2456 \mathrm{~nm}$ separated $\mathrm{Al}$ dimers and possess a $0.4 \mathrm{~nm}$ periodicity along the [00001] axis and show the same aperiodic distribution along the [10000] axis as the columns at the surface shown in Fig. 6(a). The position of these $\mathrm{Al}$ dimers with respect to the tiling is indicated by the violet arrow on the left top side of Fig. 6(c). The selected $\mathrm{Al}$ dimers are denoted in Fig. 6(c) by red squares which are positioned within the small red motifs. One of the $\mathrm{Al}$ atoms lies on the red circle whereas the other $\mathrm{Al}$ atom is the center atom of the small red motif. Since the small red motifs surround the large wheel-like blue motifs which are placed at the vertices of the tiling, we can express the aperiodic sequence of the Al dimers along the [10000] axis in terms of the fundamental tiling edge length. The intercolumnar distances which are shown to be equivalent to 
the inter-Al-dimer distance are identical to $2 \cos \left(18^{\circ}\right) \tau^{n}$ $1.979 \mathrm{~nm}$, where $1.979 \mathrm{~nm}$ is the edge length of the tiling. The arrows A (pink) and B (brown) denote the tiling edges to describe the Fibonacci units resulting for $n=-1$ into $L^{\prime} S^{\prime}$ and for $n=0$ into $L^{\prime} S^{\prime} L^{\prime}$; see also Table III. However, one has to note that only the two Fibonacci units $L^{\prime} S^{\prime}$ and $L^{\prime} S^{\prime} L^{\prime}$ between $\mathrm{Al}$ dimers were observed at the densest planes in the bulk model whereas the shorter measured intercolumnar distance $L^{\prime}$ could not be detected in the bulk model. The second match between the surface structure and the densest planes of the bulk model is the appearance of rows with low density of atoms at equivalent positions, which would show up as depressions in the STM topography. The rows with the broadest gap of atoms can be identified as the center of the large wheel-like blue motifs. Regarding the complex region between the columns one has to consider that these areas show a high variety in structure; even along the periodic [00001] axis they possess periodicities larger than the bulk structure. Although these areas are not describable a priori by the unreconstructed bulk model, bended structures or rows with high density remind us to some extent of these complex surface structures observed in the STM image of Fig. 6(a).

Several diffraction spots of the HAS and the SPA-LEED results of Sharma et al. ${ }^{11}$ can be connected with the above reported lengths between the columns of the (12110) surface. By using the same notation ${ }^{11}(m, n)$ with the relation $k_{\|}$ $=1.02 \AA^{-1}(m+n \tau)$ and the conversion from reciprocal space $\left(a^{*}\right)$ to real space $a=4 \mathrm{pi} /\left[\operatorname{sqrt}(5) a^{*}\right]$, the $L^{\prime}$ intercolumnar distance is identified as the $(2,-1)$ spot, the most frequently observed $L^{\prime} S^{\prime}$ intercolumnar distance as the $(-3,2)$ spot, and the largest segment corresponds to the $(5,-3)$ spot which was not reported by Sharma et al. ${ }^{11}$ We assumed that the amplitude of the $(5,-3)$ spot might be attenuated by the structure factor and it is included in the tail of the specular spot. We therefore conclude the importance of the sequence of the columnar structures not only for the STM imaging but also for the electron and He scattering experiments.

\section{B. Summary: The surface structure of (12110) $d$-Al-Ni-Co}

The surface structure investigation of the (12110) $d$-Al-Ni-Co has been performed in real space. In addition to the reports of the He-atom scattering experiment of Sharma ${ }^{13}$ the STM investigation and the intensive comparison to the bulk model revealed the following statements. First, both investigated QC no. 1 and QC no. 2 possess the same minimal bulk periodicity of $0.4 \mathrm{~nm}$ along the periodic axis [00001]. Second, both compositions show identical $S$ and $L$ step heights between parallel terraces which agree with the distances between the densest planes of the bulk model. Third, the most striking (12110) surface feature of both compositions, the columnar structures, are identified at the densest planes of the bulk model as rows of $\mathrm{Al}$ dimers separated by $0.2456 \mathrm{~nm}$. These Al-dimer columns possess along the aperiodic [10000] axis a sequence and intercolumnar distances which can be described by the same Fibonacci sequence as the columnar structure at the surface. The unit lengths of this Fibonacci sequence can be extracted out of

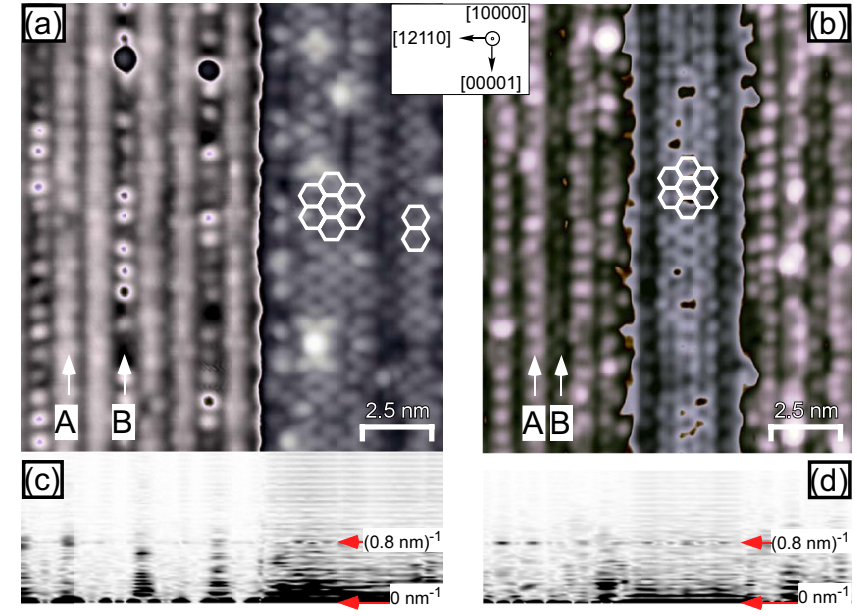

FIG. 7. (Color online) Comparison of the (10000) $d$-Al-Ni-Co surfaces. (a) STM images of QC no. 2, the (10000) facet grown on the (12110) surface, $T=5 \mathrm{~K}, I_{T}=1.5 \mathrm{nA}, \Delta z=5.15 \AA$, and $U_{T}=$ -0.5 V. (b) STM image of QC no. 3, the (10000) surface parallel to the cutting direction of the quasicrystal, $I_{T}=0.25 \mathrm{nA}, \Delta z=3.25 \AA$, and $U_{T}=+0.6 \mathrm{~V}$. (c) and (d) illustrate the calculated row-by-row FFT along the periodic [00001] axis of the according STM images.

the fundamental pentagonal Penrose tiling with a $1.979 \mathrm{~nm}$ edge length. The comparison between two different compositions of QC no. 1 and QC no. 2 shows also that next to the above described similarities the surface fine structure is slightly different. The largest distinction is found in the fine structures of the columns and in the complex area between the columns. This indicates that for both compositions, the frameworks, where the main features of the surface structure are attached, are identical and possess the same characteristic tiling length of the bulk models ${ }^{15,28}$ but the formation of the surface fine structure shows a composition dependence and a surface reconstruction in comparison to the bulk model.

\section{Surface structure of (10000) $d$-Al-Ni-Co}

The faceting of the (12110) surface into planes with $\{12110\}$ and $\{10000\}$ orientation enables the investigation of two different surfaces, the (12110) and the (10000) surface at once for the same crystal composition. Also the aperiodic sequence perpendicular to the (10000) surfaces can be investigated since besides the faceting also plane-parallel (10000) surfaces separated by a well defined terrace step exist. Before describing in detail the (10000) surface structures we will demonstrate that the (10000) surface grown on the faceted (12110) surface on QC no. 2 shows basically the same surface structures as the (10000) surface of QC no. 3. In contrast to the discussion above about the (12110) surface both compositions QC no. 1 and QC no. 2 reveal an identical surface structure for the (10000) surface. Therefore, we can disregard in this discussion the influence of the composition to the (10000) surface structure.

Figure 7 presents a comparison of the (10000) $d$-Al-Ni-Co surface structures of QC no. 2 (a) and QC no. 3 (b). Both STM images shown in Figs. 7(a) and 7(b) display neighboring terraces, where for each single terrace the contrast is 
adjusted and maximized. Figure 7(a) shows two neighboring but structural inequivalent terraces. On the left-hand side of Fig. 7(a) a terrace with a strong columnar motif along the periodic [00001] axis can be observed. This so-called row terrace exhibits two different columnar structures, which are labeled $\mathrm{A}$ and $\mathrm{B}$. The $\mathrm{A}$ rows have a significantly denser appearance than the B rows. Both rows show a pronounced $0.8 \mathrm{~nm}$ periodicity as shown by the row-by-row FFT image presented in Fig. 7(c). On the right-hand side of Fig. 7(a) a terrace possessing rows with a quasihexagonlike structure is presented. This so-called hex terrace shows groups of three or less frequently single hexagonal-like rows also with a periodicity of $0.8 \mathrm{~nm}$, as shown in Fig. 7(d). Having presented the key structure features of the faceted (10000) surface we will compare it to the terraced (10000) surface. The STM image of the (10000) surface in Fig. 7(b) shows three terraces, in the middle a so-called hex terrace and to the left and right two so-called row terraces. It is evident that the (10000) surface structure of QC no. 3 is basically identical to the (10000) surface structure of QC no. 2 and all structure features can be recovered. For instance the row terrace presents both types of rows A and B as well as the hex terrace shows the prominent triple hexagonal-like row as indicated in Fig. 7(c). This observation demonstrates that the (10000) facet grown on the (12110) surface of QC no. 2 possesses the same surface structure as the (10000) surface which lay parallel to the cutting direction of QC no. 3.

In the following, a comparative study of the step height sequence between (10000) terraces of QC no. 2 and QC no. 3 is performed. Figure 8 (a) presents the line profiles taken along the row terraces and hex terraces for both QCs. On the left-hand side the line profile along QC no. 3 (terraced) and on the right-hand side along QC no. 2 (faceted) is shown. The colored lines on the STM images in the upper part of Fig. 8(a) denote the positions of the extracted line profiles. Both QCs possess the same $L^{*}=0.78 \AA$ step height going from the hex terrace to the row terrace which is indicated by the dashed lines and by the pink rectangles at the right-hand side. The corrugation of the terraces, which will be discussed in detail below, is directly visible in the line profile and is in the range of $S^{*}$. Also larger steps heights than $L^{*}$ (not shown) of QC no. 2 and QC no. 3 do agree with each other. Hence we conclude that no distinction between the two QC surfaces is necessary.

The surface corrugations of the two different terraces types are analyzed by their height frequency distribution. Figure 8(b) shows in the upper panel the height frequency distribution of the row terrace and in the lower panel the hex terrace. The measured height-frequency distribution is fitted by a double Gauss fit where for illustrative purposes the two single Gaussians are shown. The bimodal height distribution for both terrace types indicates that the terraces do not represent single flat atomic terminations, but consist of puckered layers, i.e., two atomic planes with small separation. The separation of these two apparent planes along the [10000] axis is for both surface structure identical and is equivalent to the second smallest interplane distance of the model with $S^{*}=0.471 \AA$. In this respect the proposed modified Bravais rule by Papadopolos, ${ }^{29}$ where a thin layer of two to three bulk planes are considered as a single termination seems to
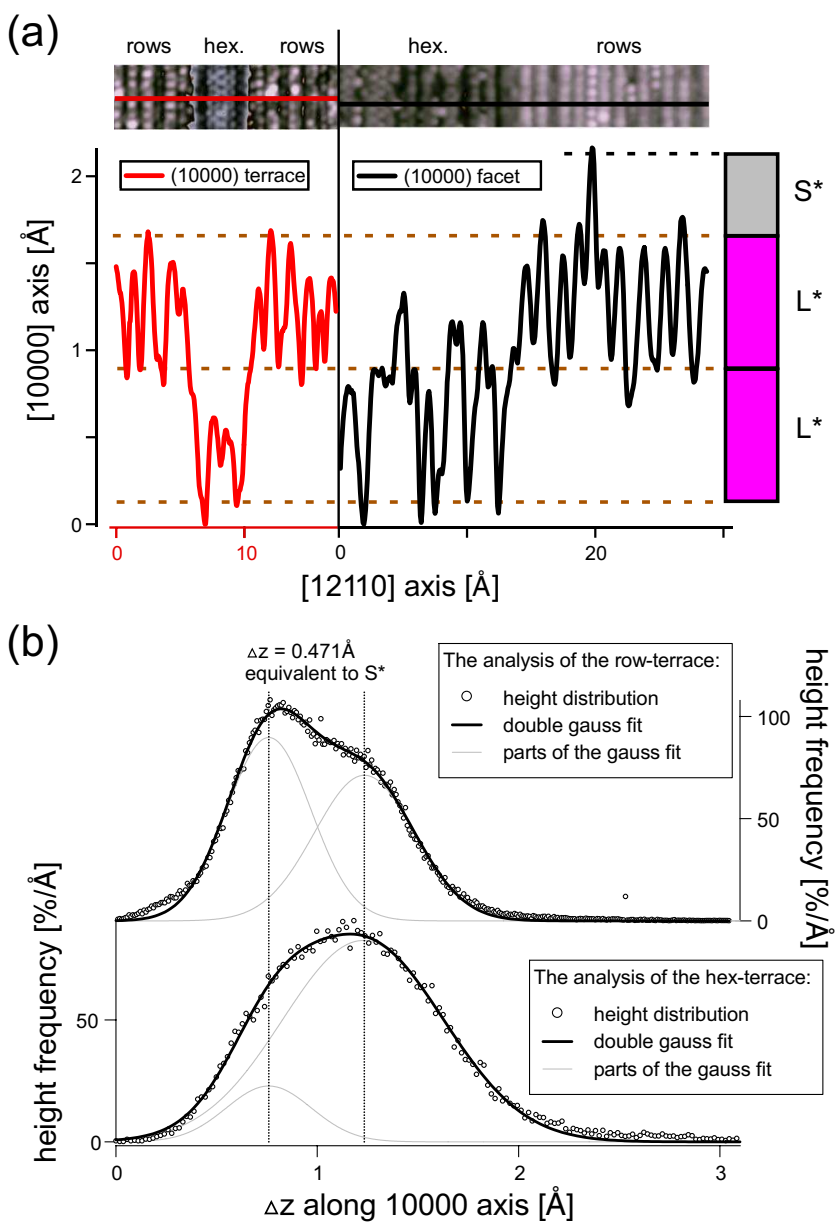

FIG. 8. (Color online) Illustration of the $d$-Al-Ni-Co (10000) step heights. (a) shows two line profiles taken along the lines in the upper STM images of QC no. 3 (terraced) and QC no. 2 (faceted), respectively. (b) shows the height frequency distribution of the row terrace in the upper panel and the hex terrace in the lower panel.

be a very reasonable approach to describe the puckered (10000) $d$-Al-Ni-Co surface. This argumentation is analogous to the description of Unal et al. ${ }^{10}$ where the terminations of the fivefold $i$-Al-Pd-Mn surface are considered to consist of two bulk planes.

In Fig. 9(a) a line profile taken along a highly terraced (10000) surface area is presented. It shows that the sequence of the step heights along the [10000] axis is consistent with a Fibonacci sequence based on the smallest interplane distance of the bulk model of $0.289 \AA$. The larger step heights on the right-hand side of Fig. 9(a) show a $L L S L$ sequence with $L$ $=(3+5 \tau) S^{*}$ which presents a long sequence of these short segments. Figure 9(b) displays a quantitative analysis of the observed step heights along the [10000] axis in comparison to the published step heights of Kishida et al., ${ }^{14}$ Park et al. ${ }^{4}$ and the distances between single planes of the bulk structure model. ${ }^{15}$ For each measured single step height between the (10000) terraces the arithmetic mean value and the corresponding frequency are calculated and illustrated in Fig. 9(b) where additionally the standard deviations are horizontally indicated. The smallest measured layer distance along the [10000] axis $S^{*}=0.469 \AA$ as well as the smallest step height $L^{*}$ and the following larger $L^{*} S^{*}$ step heights are labeled. For 
(a)

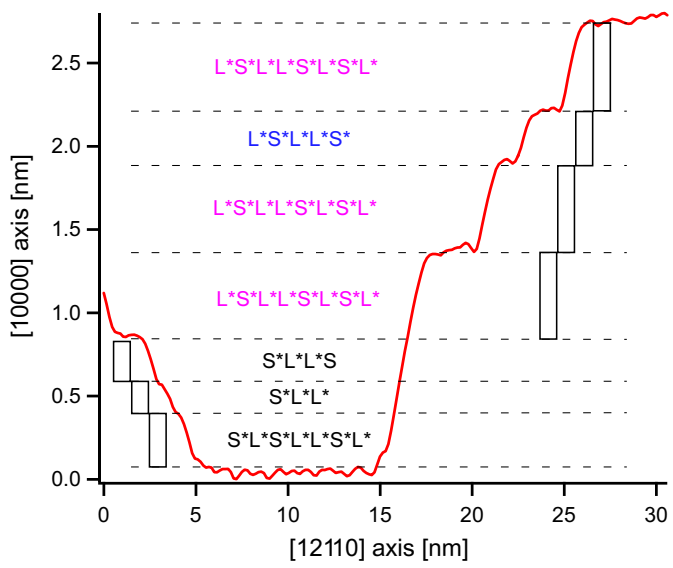

(b)

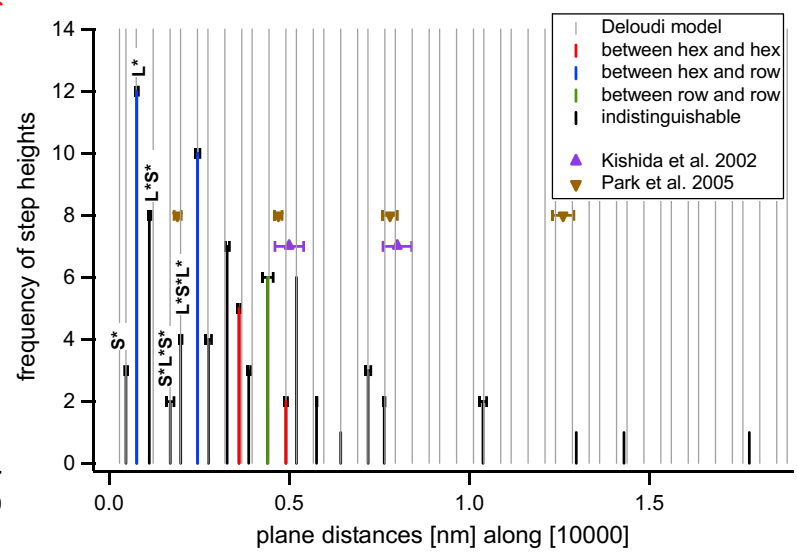

FIG. 9. (Color online) (a) Line profile taken along the [12110] axis of a highly terraced (10000) surface. The step heights are indicated by black rectangles and dashed lines and are labeled by the corresponding Fibonacci sequence. (b) The frequency of single step heights between (10000) surfaces compared to the interplane distances of the bulk model and published results.

some step heights the involved terraces types could be determined, which are colored in Fig. 9(b). For instance two different step heights between a hex terrace and a row terrace are denoted by a blue vertical line. The black vertical lines indicate that the resolution on the involved layers was too low to clearly distinguish between a row terrace and a hex terrace. In general the arithmetic mean value of the measured single step heights agrees well with the interplane distances of the bulk model and most of the smallest interplane distances were detected. In this large variety of possible and small step heights along the [10000] direction also the published step heights of Kishida and Park, shown with an arbitrary offset along the frequency axis, fit within the specified measurement error into the structure model. However, it is evident that with a typical plane separation on the order of 25-47 p.m. the correspondence of the model distances with the experiment is within the experimental error for large step heights above $1 \mathrm{~nm}$.

In the following, a detailed discussion on the (10000) $d$-Al-Ni-Co surface structure is given. The first analysis takes the bias sensitivity of the STM experiment into account and shows the result of a tentative atom assignment to highresolution STM images. Figure 10 displays in the upper part the so-called hex terrace and in the lower part the row terrace of the (10000) $d$-Al-Ni-Co surface. The two STM images on the left-hand side are recorded at $-0.5 \mathrm{~V}$ sample bias, which represents the integrated occupied density of states (DOS) from the Fermi level to $-0.5 \mathrm{~V}$. Likewise, the right-hand side of Fig. 10 presents the unoccupied DOS $(+0.5 \mathrm{~V})$ recorded simultaneously. The row terrace shows hardly any difference between the two imaging modes. The only distinction is a slightly higher contrast on the A rows for the occupied DOS representation. A tentative assignment to possible single atom positions is performed and indicated by black circles with a diameter of $2 \AA$. The resulting picture shows that an A row consists of stacked atom dimers whereas a B row rather consists of a chain of single atoms, both with a $0.8 \mathrm{~nm}$ periodicity along the periodic [00001] axis. Along the aperiodic [12110] axis the interatomic distances can be described with lengths following the Fibonacci series with $L^{\prime \prime}$
$=0.467 \mathrm{~nm}$ (taken from the bulk model), which fits to the interatomic distance within a dimer on the A row. On the other hand the hex terrace shows significant differences between the unoccupied and occupied DOS images. The STM image representing the unoccupied $(+0.5 \mathrm{~V})$ DOS shows three rows with a hexagonal-like pattern. However, when recording the occupied DOS the same location shows rather a columnar shape where the hexagonal-like pattern is only weakly visible. Nevertheless, for both imaging modes a common tentative assignment for the hex-terrace to single atom positions can be performed. For the shown sketch in Fig. 10 a quasihexagonal subunit is used. This subunit has a $0.8 \mathrm{~nm}$ periodicity along the periodic [00001] axis. The short length of the hexagon subunit along the aperiodic axis is determined
$-0.5 \mathrm{~V}$

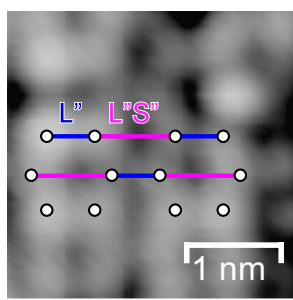

[12110]

-

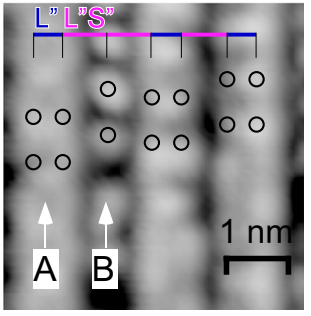

$+0.5 \mathrm{~V}$

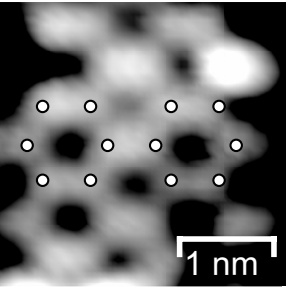

[0001]

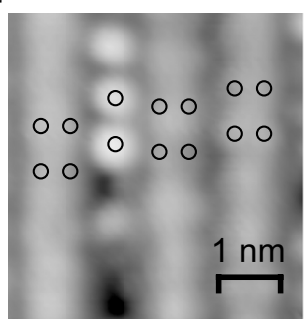

FIG. 10. (Color online) High-resolution STM images of the (10000) row and hex terrace $d$-Al-Ni-Co of QC no. 2 surface. On the left-hand side STM images recorded at $-0.5 \mathrm{~V}$ presenting the occupied DOS and on the right-hand side the STM images showing the unoccupied DOS $(+0.5 \mathrm{~V})$. A tentative atom assignment is indicated by circles. 


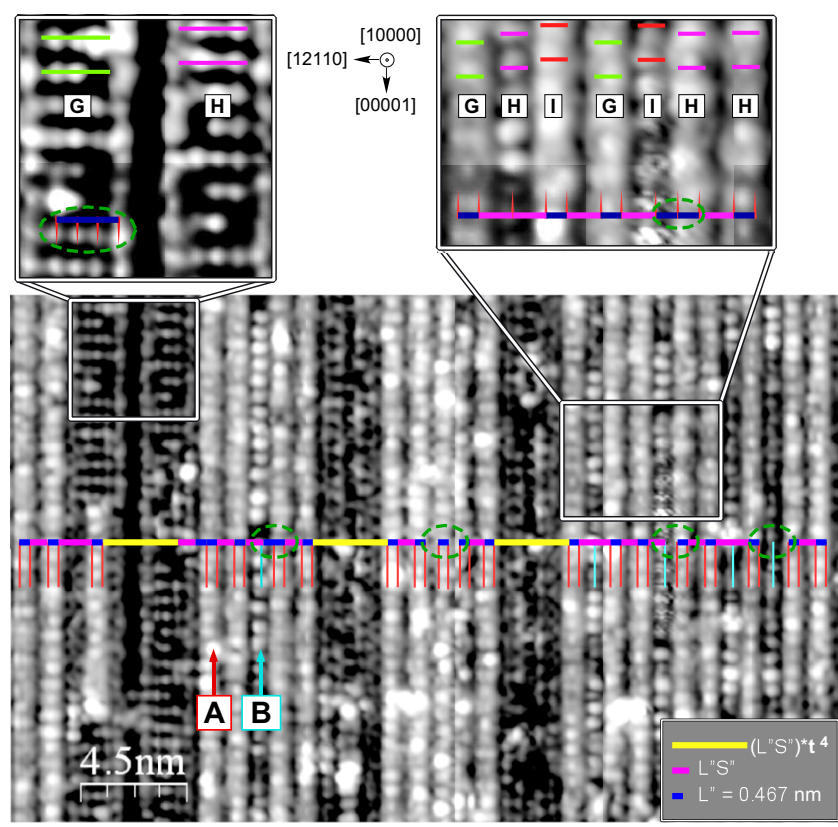

FIG. 11. (Color online) STM image of the row terrace of the (10000) $d-\mathrm{Al}_{72.9} \mathrm{Co}_{16.7} \mathrm{Ni}_{10.4}(\mathrm{QC}$ no. 2$)$ surface recorded at $5 \mathrm{~K}$, $I_{T}=0.7 \mathrm{nA}, U_{T}=0.1 \mathrm{~V}$, and $\Delta z=2.9 \AA$. The sequence of the bright rows is indicated by units of a Fibonacci sequence which is distorted at the positions of the green dashed circles. The upper left panel shows a zoom in of the lower lying plane. In the upper right panel a zoom in of the upper lying plane is shown where $0.4 \mathrm{~nm}$ periodic layers are indicated by the letters G, H, and I.

to be $L^{\prime \prime}=0.467 \mathrm{~nm}$ whereas the long length is equivalent to $L^{\prime \prime} S^{\prime \prime}$, which are the same Fibonacci building blocks as we used for the row terrace.

To investigate the aperiodic sequence of the main structure motifs on the (10000) $d$-Al-Ni-Co surface highresolution and large area STM images of the row and hex terraces are presented and discussed. As mentioned above we assume both (10000) terrace types to consist of two atomic planes separated by $S^{*}=0.471 \AA$, which might be regarded a single puckered layer. Figure 11 shows an STM image of the row terrace of the (10000) facet recorded on QC no. 2. The main structure motifs of the row terrace, the A and B rows, are present in the upper layer (brighter). The sequence of the tentatively assigned atoms on the A and B rows along the aperiodic axis [12110] is denoted by vertical red lines for atoms belonging to $\mathrm{A}$ rows and turquoise lines for atoms belonging to $\mathrm{B}$ rows. The interatomic distances for dimers at the A rows are equivalent to $L^{\prime \prime}=0.467 \mathrm{~nm}$ and are indicated with blue horizontal lines. The distances between a B row and an A row or between two A rows are equivalent to $L^{\prime \prime} S^{\prime \prime}=0.756 \mathrm{~nm}$ and are indicated with pink horizontal lines. At places where the upper layer is not continuous a long yellow line equivalent to $\left(L^{\prime \prime} S^{\prime \prime}\right) \tau^{4}$ is used to indicate a hypothetical undisturbed Fibonacci sequence. By analyzing the sequence of the inter-row distances it turns out that only fragments of a Fibonacci sequence are present. The distortions from a perfect Fibonacci sequence are denoted by dotted green circles. The top right panel in Fig. 11 shows a zoom in of the upper layer where in detail the sequence of the $\mathrm{A}$ and $\mathrm{B}$ rows is visible. The indicated sequence breaks

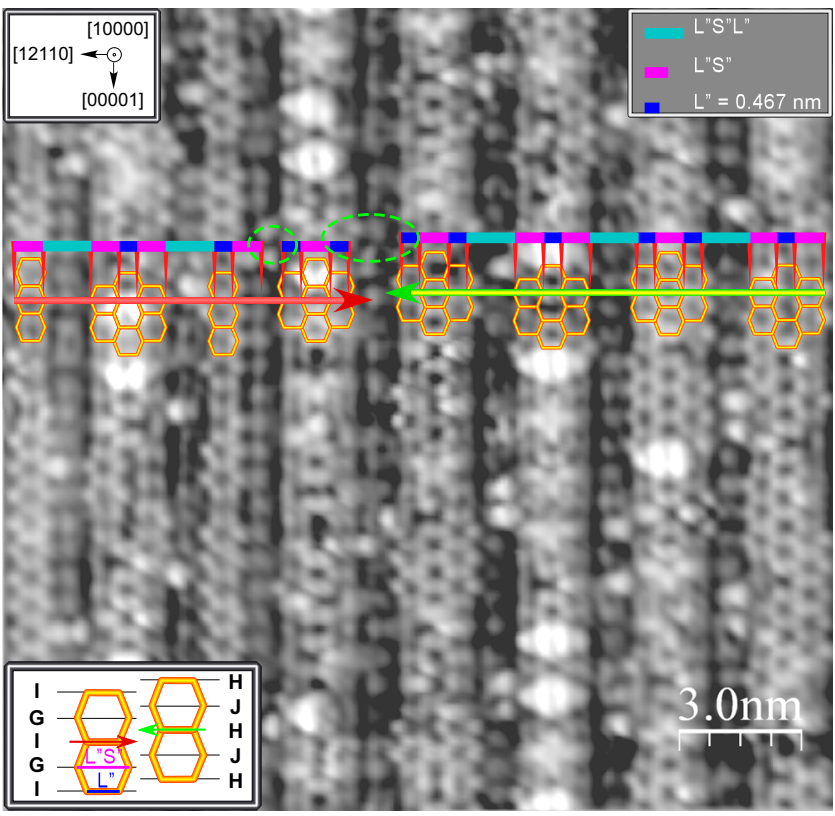

FIG. 12. (Color online) STM image of the so-called hex terrace of the $d-\mathrm{Al}_{72.9} \mathrm{Co}_{16.7} \mathrm{Ni}_{10.4}(\mathrm{QC}$ no. 2) (10000) surface recorded at 5 $\mathrm{K}, I_{T}=0.05 \mathrm{nA}, U_{T}=0.5 \mathrm{~V}$, and $\Delta z=3.1 \AA$. Two different $0.8 \mathrm{~nm}$ periodic columns are apparent: a single and a triple hexagonlike columns. Along the aperiodic axis [12110] the sequence is indicated by Fibonacci units derived from the applied bulk model. The sequence is distorted at two green circles.

the law of forming a true Fibonacci sequence since two $S^{\prime \prime}$ lengths follow one after the other, denoted by a green circle. The second feature observable on the top right panel is that the rows are shifted with respect to each other along the periodic [00001] axis. These shifts are marked by green, pink, and red horizontal lines and are additionally labeled by letters. Rows with the same position along the [00001] axis are indicated with the same color and the same letter. The shift between the rows $\mathrm{G}$ and $\mathrm{H}$ or $\mathrm{H}$ and I along the [00001] is $0.2 \mathrm{~nm}$, which is equivalent to the interplane distance of the bulk model. However, the description of the lower lying plane is more difficult. Figure 11 reveals three large and dark vertical stripes. The two stripes on the right-hand side reveal a surface structure which reminds us of the hex terrace. But the stripe on the left-hand side shows a new motif. The zoom in on the upper left-hand side presents this new motif in detail. At first sight the surface structure reminds us of two $0.8 \mathrm{~nm}$ periodic ladder structures. Both ladders are shifted with respect to each other along the [00001] axis by $0.2 \mathrm{~nm}$ which is denoted by the green and pink horizontal lines and labeled with the letters $G$ and $H$. The sequence within a single step of the ladders which is indicated by three $L^{\prime \prime}$ Fibonacci segments presents therefore rather a periodical order of this ladderlike surface, as far as this can be determined from only a short structural sequence.

The STM image recorded at $+0.5 \mathrm{~V}$ shown in Fig. 12 is displayed as a representative surface to discuss the hex terrace in detail. The surface presents clearly the periodic quasihexagonal row structure along the [00001] axis. The hexagonal character of these rows is indicated by yellow hexagons. Two different kinds of columns are observed: some columns 
exhibiting only one row of hexagons and, more frequently, columns presenting a triple row of hexagons combined to a columnar honeycomb structure are found. The aperiodic sequence in the [12110] direction is analyzed along the red and green arrows and denoted by blue $L^{\prime \prime}$ and pink $L^{\prime \prime} S L^{\prime \prime}$ bars with $L^{\prime \prime}=0.467 \mathrm{~nm}$. The deviations from a perfect Fibonacci sequence are indicated by two green dashed ellipses. The most striking deviation is denoted by the larger of these in the middle of Fig. 12 where the two red and green arrows indicate a $0.2 \mathrm{~nm}$ vertical shift between the honeycomblike columns along the [00001] axis. The lower left inset in Fig. 12 schematically illustrates this shift between the hexagonal subunit structures. The shift along the [00001] axis of $0.2 \mathrm{~nm}$ means that the hexagonal structures have their vertices on two complementary layers along the [00001] axis indicated either with the letters I and $\mathrm{G}$ or $\mathrm{H}$ and J. These would now correspond to $\mathrm{B}$ and $\mathrm{D}$ layers (or A and $\mathrm{C}$, respectively) in the bulk structure. As yet, it is still unclear whether this shift is due to a defect in the surface structure, e.g., a line defect or if there is a possible bulk defect leading to this slip from the $\mathrm{A} / \mathrm{C}$ plane group to the $\mathrm{B} / \mathrm{D}$ group.

Another feature of the hex terrace is that some protrusions are present which are located at the center of the triple hexagonlike column whereas the single hexagonlike columns remain undecorated. Likewise to the row terrace also the hex terrace presents a double plane structure. But the hex terrace shows more clearly the upper plane structure with the hexagonlike columns and only a $0.8 \mathrm{~nm}$ periodic row between the hexagonlike columns is present on the lower terrace.

Figure 13(a) illustrates a small section of the bulk model ${ }^{15}$ viewed along the [00001] axis, where at the center of the tiling vertex the blue circle denotes a large wheel-like motif which is surrounded by ten small pentagonal and decagonal motifs indicated by red circles. In contrast to the illustration of the model structure in Fig. 6(c) the atom positions are now labeled according to their associated layers along the [00001] axis. The two lines A and B in Fig. 13(a) represent hypothetical (10000) surface termination cuts. It can be observed that the (10000) cut is selective in respect of the layer types. The line A cuts only through the atoms of layers $A$ and $C$ whereas the dashed line B cuts only through the layers B and D. This is a general property of (10000) plane terminations which leads to minimal interplane distances of $0.4 \mathrm{~nm}$ along the periodic [00001] axis. However, the minimal measured distance along the periodic [00001] axis between rows on the row terrace (Fig. 11) and between the hexagonal rows on the hex terrace (Fig. 12) is only $0.2 \mathrm{~nm}$.

The Fibonacci basis lengths $L^{\prime \prime}$ and $L^{\prime \prime} S^{\prime \prime}$ to describe the aperiodic sequence between the rows in Fig. 11 or between the hexagonlike rows in Fig. 12 are taken from the diagonal distance of the pentagon with an edge length of $0.287 \mathrm{~nm}$ and the $\tau$ inflated pentagon, respectively. Both pentagons and basis lengths are indicated in the middle of Fig. 13(a). A short aperiodic sequence equivalent to those used in Figs. 11 and 12 consisting of $L^{\prime \prime}$ and $L^{\prime \prime} S^{\prime \prime}$ Fibonacci units is denoted along one line. Figure 13(b) illustrates for both orientations the minimal experimentally observed step height referring to the structure model. For the (10000) surfaces a very small layer distance and very small step heights are measured in-

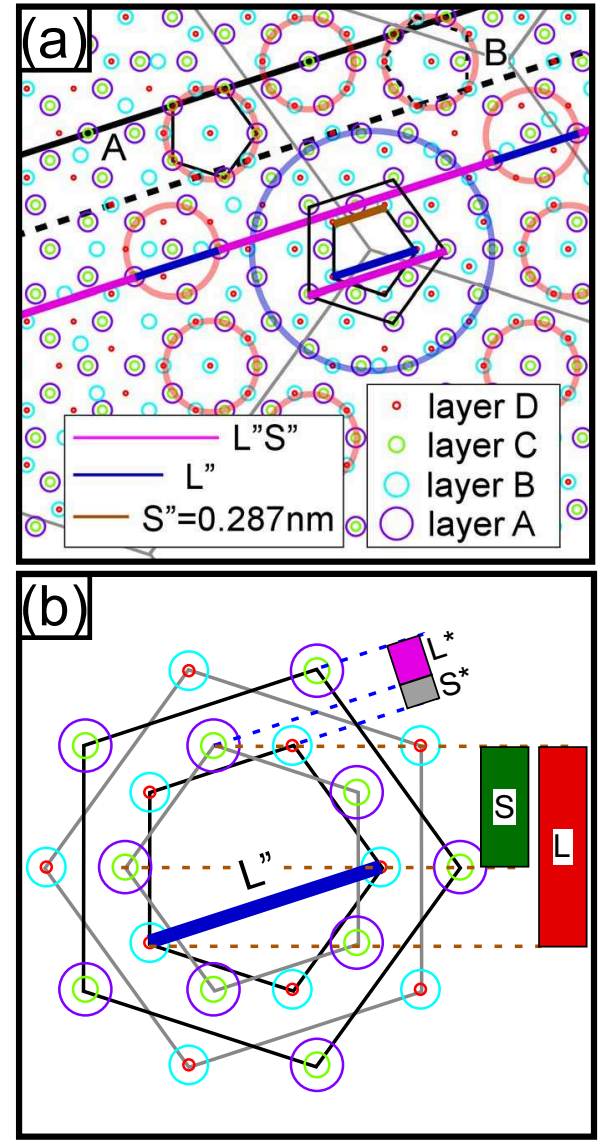

FIG. 13. (Color online) (a) Tenfold view of the Co-rich Al$\mathrm{Ni}$-Co bulk model. The atom positions are labeled according to their positions along the tenfold direction. The unit lengths of the derived Fibonacci sequence to describe the aperiodic sequence within the (10000) surfaces are equivalent to the diagonal distances of the two indicated pentagons. Two different (10000) cuts are represented by the lines A and B. (b) Illustration of the minimal measured step heights for both orientations. The minimal measured (10000) interlayer distance indicated by $S^{*}$ is very small compared to the minimal observed (12110) step height denoted by $S$.

dicated by $S^{*}$ and $L^{*}$ in contrast to the (12110) surface where larger step heights are recorded indicated by $S$ and $L$. Otherwise to the selected cut of the (10000) layers, all four layers are present on a (12110) surface which is denoted by the dashed lines in Fig. 13(b).

In comparison with the different published reports on the (10000) $d$-Al-Ni-Co twofold surface, ${ }^{4-6,11,12,14}$ the STM investigations of Park et al. ${ }^{4}$ and Kishida et al. ${ }^{14}$ and the HAS and SPA-LEED reports of Sharma et al. ${ }^{11}$ are in the context of this paper the most relevant articles. We can conclude that the surface structure of the (10000) Co-rich $d$-Al-Ni-Co presented here reminds us in general of the findings of Kishida et $a l .{ }^{14}$ On the other hand little similarities with the surface structure of Park et al. ${ }^{4}$ could be discovered. The (10000) $d-\mathrm{Al}_{72} \mathrm{Ni}_{11} \mathrm{Co}_{17}$ surface structure presented by Park et al. ${ }^{4}$ was prepared identically compared to the here presented (10000) $d-\mathrm{Al}_{72.9} \mathrm{Co}_{16.7} \mathrm{Ni}_{10.4}$ surfaces and both compositions are almost equivalent. Park et al. ${ }^{4}$ report that only 10\%-20\% of the surface shows rows with a $0.8 \mathrm{~nm}$ periodicity whereas the here presented surface shows a clearly $0.8 \mathrm{~nm}$ periodic- 
ity. On the other hand similar spacing between columns along the aperiodic [12110] axis and some of the observed step heights are within the measure error equivalent to the surface structure of Park et $a l .{ }^{4}$ In spite of some similarities the surface morphology appears different. On the other hand the surface morphology of Kishida et al. ${ }^{14}$ seems to be related to the so-called row structure presented here. Kishida et al. ${ }^{14}$ reported some faint, remnant $0.4 \mathrm{~nm}$ periodicity along the A rows which could not be observed by us. The aperiodic sequence of the rows could not be compared to each other since Kishida et al. $^{14}$ did not report it and it was even assumed that Kishida's surface shows a periodic sequence. ${ }^{4}$

The two Fibonacci lengths $L^{\prime \prime}$ and $L^{\prime \prime} S^{\prime \prime}$ along the [12110] aperiodic axis of the (10000) surface were compared to the HAS and SPA-LEED results of Sharma et al. ${ }^{11}$ With the relation $k_{\|}=1.19 \AA^{-1}(m+n \tau)$ (using the basic basis, $b \chi$ $=1.19$ from Ref. 11) and the conversion from reciprocal space $\left(a^{*}\right)$ to real space $a=4 \mathrm{pi} /\left[\operatorname{sqrt}(5) a^{*}\right]$, the $L^{\prime \prime}$ length is identified as the $(1,0)$ spot and the $L^{\prime \prime} S^{\prime \prime}$ length corresponds to the $(-1,1)$ spot. This indicates the relevance of the row and hex surface structures we have identified here for the electron and He scattering.

\section{Summary: The surface structure of (10000) $d$-Al-Ni-Co}

The surface analysis of the (10000) $d$-Al-Ni-Co quasicrystal revealed interesting aspects. We could show that the (10000) surface, which is present on the faceted surface of the (12110) oriented $d$-Al-Ni-Co, exhibits an identical surface structure as the surface of a (10000) oriented $d$-Al-Ni-Co quasicrystal. The detailed (10000) surface analysis revealed that two different parallel terraces exist, the socalled row terrace and the so-called hex terrace. The analysis of the height distribution on each terrace type revealed that they consist of two planes separated by $S^{*}=0.471 \AA$. This finding indicates that the (10000) surfaces do not consist of a single dense plane but rather of puckered layers consisting of two low density planes which present the surface termination. The step heights between the (10000) surfaces agree also well with the plane distances of the bulk model. In contrast to the row terrace the hex terrace shows significant difference in the occupied and unoccupied electronic DOS recorded by STM at negative and positive sample bias, respectively. The main structure motif of the row terrace are two different columns along the [00001] axis which are allocated to a dimer row and a single chain of atoms. The hex terrace presents rows with a columnar quasihoneycomb structure when probing the unoccupied DOS. The row terrace as well as the hex terrace present a columnar structure with smallest periodicity of $0.8 \mathrm{~nm}$ along the [00001] axis. However, on both (10000) surface types the columns are shifted with respect to each other along the periodic [00001] axis by $0.2 \mathrm{~nm}$. This $0.2 \mathrm{~nm}$ shift cannot be explained by a single plane of the bulk model, since single planes which represent the (10000) surface of the bulk model possess a minimal interlayer distance of $0.4 \mathrm{~nm}$ along the [00001] axis. On the other hand, the fragmentary but equivalent aperiodic sequence along the [12110] axis of both terrace types follows a Fibonacci sequence based on the edge length of the second smallest pentagon from the bulk structure model with $L^{\prime \prime}$ $=0.467 \mathrm{~nm}$.

\section{E. Differences between the (12110) and (10000) $d$-Al-Ni-Co surfaces}

In this last part we discuss the most relevant differences between the structures of the two twofold $d$-Al-Ni-Co surfaces and relate them to the bulk structure. One major difference is that the (10000) terraces show a bimodal height distribution inferring that it consists of two different well defined planes with a separation of only $S^{*}=0.471 \AA$, whereas the (12110) surface shows clearly a single Gaussian height distribution (not shown here) which can be allocated to a single atomic plane. The consequence of this interpretation is that each of the atomic planes which represents the (10000) surface must have a lower atomic density compared to the single plane which represents the (12110) surface. This argument on the other hand gives an indication why the observed smallest step height of the (10000) surfaces is so much smaller than the smallest step height between (12110) terraces [see Fig. 13(a)]. Therefore, the bulk structure model is analyzed regarding plane density and composition for the two orientations. Figure 14(a) shows the result of this analysis of a large cuboid bulk model with dimensions of 40 $\times 40 \times 0.8 \mathrm{~nm}^{3}$ as indicated schematically on the lower right-hand side of the figure. All planes parallel to the [12110] and parallel to the [10000] axis were analyzed in respect of their density (number of atoms per $\mathrm{nm}^{2}$ ) and composition (at.\% Al). The difference in the density of the (10000) planes in comparison to the (12110) is obvious. The densest (12110) planes of the bulk model are around 1.7 times denser than the densest planes of the (10000) orientation. From this observation one can understand that the (10000) termination presents a puckered layer of two low density bulk planes. Correspondingly, also the minimal step height is expected to be smaller for the (10000) termination. The second remarkable distinction is the correlation of the composition with the density of the (12110) planes which reaches, for the densest planes, 61 at.\% $\mathrm{Al}$ and 39 at.\% TM, which is slightly lower in $\mathrm{Al}$ than the mean composition of the structure model. On the other hand the bulk analysis of the (10000) planes reveals a very large scattering between the composition and density. This large chemical variability within the densest (10000) planes of the bulk model and the nonsystematic absence of the periodic layers along the [00001] axis on the (10000) STM images complicates very substantially the comparison between the measurement and the bulk model. A simpler situation is found by analyzing the densest planes of the (12110) bulk model. It turns out that all these planes show similar structure motifs as found on the recorded STM images, as shown in Fig. 6. The consequence that the densest planes are regarded to represent the (12110) surface is that the surface concentration is not high in $\mathrm{Al}$ but rather in the range of the bulk concentration.

To verify this conclusion the surface concentration was investigated by performing XPS experiments of the (12110) QC no. 2 surface in dependence of the enclosed angle between the surface normal and the photoelectron detector. The 


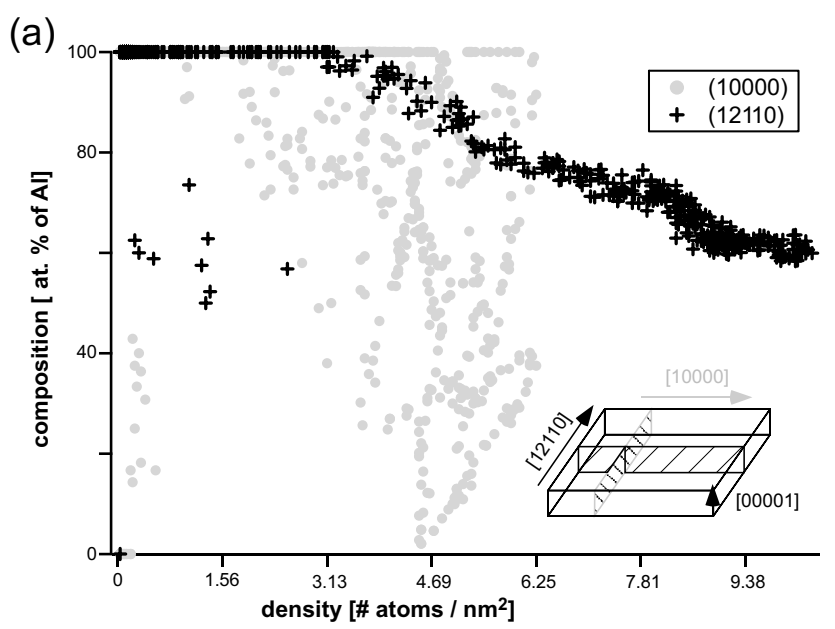

(b)

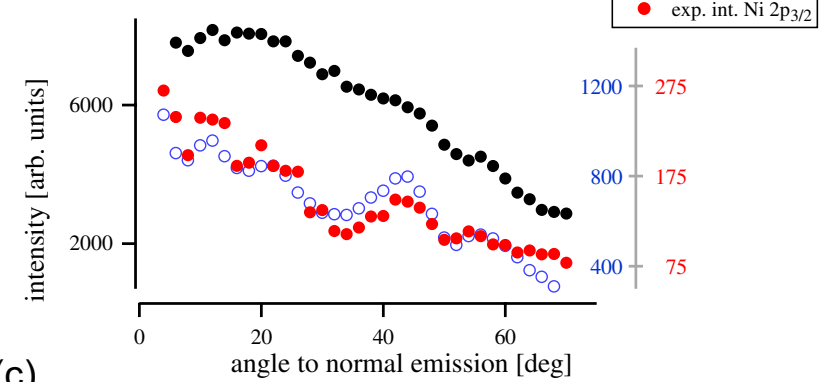

(c)

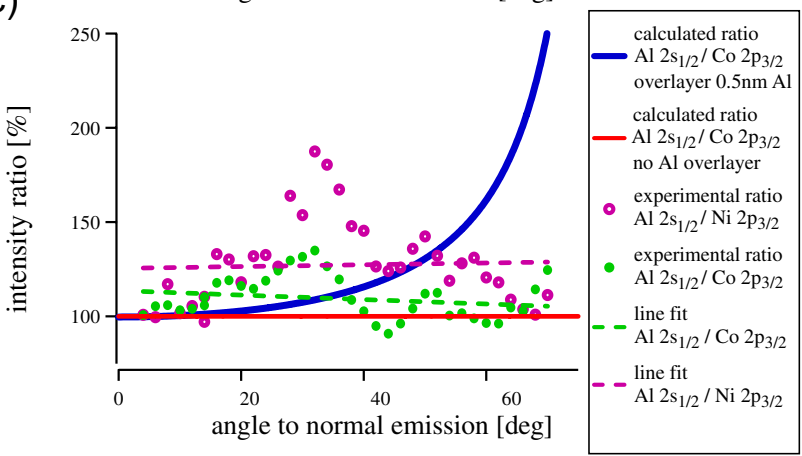

FIG. 14. (Color online) (a) Analysis of the bulk model planes. The two orthogonal planes (10000) and (12110) are analyzed in respect of their density and composition. The graph shows that the (12110) planes are by a factor of 1.7 denser as the (10000) planes. The (12110) presents additionally a correlation between the composition and the density. (b) XPS analysis in dependence of the angle to normal emission of the $d-\mathrm{Al}_{72.9} \mathrm{Co}_{16.7} \mathrm{Ni}_{10.4}$ (QC no. 2) surface along the periodic direction. (c) The ratio of the $\mathrm{Al} 2 s_{1 / 2} / \mathrm{Co} 2 p_{3 / 2}$ and $\mathrm{Al} 2 s_{1 / 2} / \mathrm{Ni} 2 p_{3 / 2}$ shows that the surface composition retains the bulk structure.

photoelectrons were collected starting from the azimuthal angle of $0^{\circ}$ (normal emission) down to $70^{\circ}$ in the direction of the periodic [00001] axis within a polar interval of $\pm 5^{\circ}$ where the intensities for the same polar angles are averaged in order to minimize effects arising from the single crystalline nature of the sample. ${ }^{30}$ Figure 14 (b) shows the intensity decrement of the $\mathrm{Al} 2 s_{1 / 2}$, Co $2 p_{3 / 2}$, and Ni $2 p_{3 / 2}$ in dependence of the azimuthal angle. The intensities were corrected by the element specific cross sections and the kinetic energy- dependent electron inelastic mean free paths. The rise in the intensity at around $40^{\circ}$ is due to the so-called forward focusing effect ${ }^{31}$ related to the single-crystal structure of the sample and is not relevant in this discussion. The calculated and normalized $\left(I=100 \%\right.$ for the azimuthal angle $\left.=0^{\circ}\right)$ ratios of the intensities of $\mathrm{Al} 2 s_{1 / 2} / \mathrm{Co} 2 p_{3 / 2}$ and $\mathrm{Al} 2 s_{1 / 2} / \mathrm{Ni} 2 p_{3 / 2}$ are illustrated in Fig. 14(c) by green and pink circles where additionally the linear fits of both ratios are indicated by dashed lines. The linear fits of both ratios reveal that the concentration does not change with decreasing probed surface depth, which is proportional to [ $\cos$ (angle to normal emission) $]^{-1}$. Two simulated intensity ratios are shown to illustrate the situation for a bulk terminated surface and a surface which is covered by an overlayer of $0.5 \mathrm{~nm}$ of $100 \%$ $\mathrm{Al}$, denoted by a red and a blue line, respectively. This comparison shows that the investigated surface retains the bulk composition to the topmost layers and does not possess an accumulation of $\mathrm{Al}$ atoms. We claim two arguments that the densest (12110) planes of the bulk model can be used to describe the (12110) surface structure. First, the main structure features of the (12110) STM images are identified only on the densest (12110) planes of the bulk model. Second, the discussed correlation between the composition and the density of the bulk model shows that planes with the bulk model composition are the densest (12110) planes, which is confirmed by the presented ARPES measurement.

\section{CONCLUSION}

The $d$-Al-Ni-Co quasicrystal exhibits the two $\{10000\}$ and $\{12110\}$ crystallographic inequivalent twofold symmetric surfaces. We investigated the (12110) surface of two different compositions $d-\mathrm{Al}_{70} \mathrm{Ni}_{15} \mathrm{Co}_{15}$ and $d-\mathrm{Al}_{72.9} \mathrm{Ni}_{10.4} \mathrm{Co}_{16.7}$ and the (10000) surface of the $d-\mathrm{Al}_{72.9} \mathrm{Ni}_{10.4} \mathrm{Co}_{16.7}$ quasicrystal by STM, SEM, and XPS. For all three surfaces, STM images presenting a columnar structure along the periodic axis are successfully obtained with atomic resolution. The investigation of both composition of the (12110) $d$-Al-Ni-Co surfaces revealed that the surface is faceted into $\{12110\}$ and $\{10000\}$ facets with a 1:1 frequency ratio. The main structure feature of the (12110) surface could be successfully allocated to the densest planes of the bulk model. The (10000) surface showed two different types of surface, the row and hex terraces. These two surface types possess structure motifs which could also be allocated to structural units of the used bulk model.

Although great efforts have been devoted here to correlate the surface structures observed in the STM with the bulk structure and attain an atomic model representation of these surfaces, this goal could not be achieved completely. Details of the origin of the $0.8 \mathrm{~nm}$ periodicity and especially of the complex surface structures with periodicity of integer multiples of $0.4 \mathrm{~nm}$ on the (12110) surface still await explanation. Also, the discrepancy to the results of Park et al. indicates that further investigation of the atomic surface structure on this highly relevant system needs to be undertaken. 


\section{ACKNOWLEDGMENTS}

The Swiss National Foundation (Contract No. SNF 200021-112333/1), the European Network of Excellence
(NoE): "Complex Metallic Alloys" CMA (Contract No. NoE 500140), and the EU-FP7: "appliCMA" project (Grant Agreement No. NMP3-SL-2008-214407) are acknowledged.
*Corresponding author; ruben.maeder@empa.ch

${ }^{\dagger}$ Present address: Van der Waals-Zeeman Institute, Universiteit van Amsterdam, 1018 XE Amsterdam, The Netherlands.

${ }^{1}$ C. Janot and J. M. Dubois, in Quasicrystals, edited by J. B. Suck, F. Schreiber, and P. Häussler (Springer, Chemnitz, 2002).

${ }^{2}$ J. Y. Park and P. A. Thiel, J. Phys.: Condens. Matter 20, 314012 (2008).

${ }^{3}$ J. Y. Park, D. F. Ogletree, M. Salmeron, R. A. Ribeiro, P. C. Canfield, C. J. Jenks, and P. A. Thiel, Phys. Rev. B 71, 144203 (2005).

${ }^{4}$ J. Y. Park, D. F. Ogletree, M. Salmeron, R. A. Ribeiro, P. C. Canfield, C. J. Jenks, and P. A. Thiel, Phys. Rev. B 72, 220201(R) (2005).

${ }^{5}$ J. Y. Park, D. F. Ogletree, M. Salmeron, R. A. Ribeiro, P. C. Canfield, C. J. Jenks, and P. A. Thiel, Phys. Rev. B 74, 024203 (2006).

${ }^{6}$ J. Y. Park, D. F. Ogletree, M. Salmeron, R. A. Ribeiro, P. C. Canfield, C. J. Jenks, and P. A. Thiel, Science 309, 1354 (2005).

${ }^{7}$ J. Y. Park, D. F. Ogletree, M. Salmeron, C. J. Jenks, P. A. Thiel, J. Brenner, and J. M. Dubois, J. Mater. Res. 23, 1488 (2008).

${ }^{8}$ R. Widmer, O. Groening, P. Ruffieux, and P. Groening, Philos. Mag. 86, 781 (2006).

${ }^{9}$ Z. Papadopolos, G. Kasner, J. Ledieu, E. J. Cox, N. V. Richardson, Q. Chen, R. D. Diehl, T. A. Lograsso, A. R. Ross, and R. McGrath, Phys. Rev. B 66, 184207 (2002).

${ }^{10}$ B. Unal, C. J. Jenks, and P. A. Thiel, Phys. Rev. B 77, 195419 (2008).

${ }^{11}$ H. R. Sharma, K. J. Franke, W. Theis, A. Riemann, S. Folsch, P. Gille, and K. H. Rieder, Phys. Rev. B 70, 235409 (2004).

${ }^{12}$ H. R. Sharma, K. J. Franke, W. Theis, A. Riemann, S. Folsch, K. H. Rieder, and P. Gille, Surf. Sci. 561, 121 (2004).

${ }^{13}$ H. R. Sharma, W. Theis, P. Gille, and K. H. Rieder, Surf. Sci. 511, 387 (2002).
${ }^{14}$ M. Kishida, Y. Kamimura, R. Tamura, K. Edagawa, S. Takeuchi, T. Sato, Y. Yokoyama, J. Q. Guo, and A. P. Tsai, Phys. Rev. B 65, 094208 (2002).

${ }^{15}$ S. Deloudi and W. Steurer, Philos. Mag. 87, 2727 (2007).

${ }^{16}$ E. Rotenberg, W. Theis, and K. Horn, Prog. Surf. Sci. 75, 237 (2004).

${ }^{17}$ S. Ritsch, C. Beeli, H. U. Nissen, T. Godecke, M. Scheffer, and R. Luck, Philos. Mag. Lett. 78, 67 (1998).

${ }^{18}$ P. Canfield and Z. Fisk, Philos. Mag. B 65, 1117 (1992).

${ }^{19}$ I. R. Fisher, M. J. Kramer, Z. Islam, A. R. Ross, A. Kracher, T. A. Wiener, M. J. Sailer, A. I. Goldman, and P. Canfield, Philos. Mag. B 79, 425 (1999).

${ }^{20}$ I. Horcas, R. Fernandez, J. M. Gomez-Rodriguez, J. Colchero, J. Gomez-Herrero, and A. M. Baro, Rev. Sci. Instrum. 78, 013705 (2007).

${ }^{21}$ S. Katrych and W. Steurer, Z. Kristallogr. 219, 606 (2004).

${ }^{22}$ W. Steurer, Z. Kristallogr. 219, 391 (2004).

${ }^{23}$ Z. Zhang and Y. Zhuang, Philos. Mag. Lett. 65, 203 (1992).

${ }^{24}$ M. Shimoda, J. Q. Guo, T. J. Sato, and A. P. Tsai, Surf. Sci. 454-456, 11 (2000).

${ }^{25}$ Y. Yan and R. H. Wang, J. Mater. Res. 8, 286 (1993).

${ }^{26}$ C. Herring, Phys. Rev. 82, 87 (1951).

${ }^{27}$ Z. Papadopolos, O. Groening, and R. Widmer, in Models, Mysteries and Magic of Molecules, edited by J. C. A. Boeyens and J. F. Ogilvie (Springer, Dordrecht, 2008).

${ }^{28}$ W. Steurer, T. Haibach, B. Zhang, S. Kek, and R. Lück, Acta Crystallogr., Sect. B: Struct. Sci. 49, 661 (1993).

${ }^{29}$ Z. Papadopolos, P. Pleasants, G. Kasner, V. Fournee, C. J. Jenks, J. Ledieu, and R. McGrath, Phys. Rev. B 69, 224201 (2004).

${ }^{30}$ C. Westphal, Surf. Sci. Rep. 50, 1 (2003).

${ }^{31}$ R. Fasel, P. Aebi, R. G. Agostino, D. Naumovic, J. Osterwalder, A. Santaniello, and L. Schlapbach, Phys. Rev. Lett. 76, 4733 (1996). 